\title{
Krylov implicit integration factor methods for spatial discretization on high dimensional unstructured meshes: Application to discontinuous Galerkin methods
}

\author{
Shanqin Chen ${ }^{\mathrm{a}, 1}$, Yong-Tao Zhang ${ }^{\mathrm{b}, *, 2}$

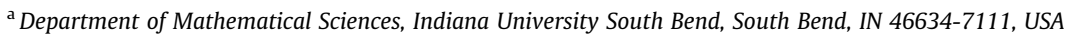 \\ ${ }^{\mathrm{b}}$ Department of Applied and Computational Mathematics and Statistics, University of Notre Dame, Notre Dame, IN 46556, USA
}

\section{A R T I C L E I N F O}

\section{Article history:}

Available online 27 January 2011

\section{Keywords:}

Discontinuous Galerkin finite element methods

Implicit integration factor methods

Krylov subspace approximation

Triangular meshes

Morphogenesis

\begin{abstract}
A B S T R A C T
Integration factor methods are a class of "exactly linear part" time discretization methods. In [Q. Nie, Y.-T. Zhang, R. Zhao, Efficient semi-implicit schemes for stiff systems, Journal of Computational Physics, 214 (2006) 521-537], a class of efficient implicit integration factor (IIF) methods were developed for solving systems with both stiff linear and nonlinear terms, arising from spatial discretization of time-dependent partial differential equations (PDEs) with linear high order terms and stiff lower order nonlinear terms. The tremendous challenge in applying IIF temporal discretization for PDEs on high spatial dimensions is how to evaluate the matrix exponential operator efficiently. For spatial discretization on unstructured meshes to solve PDEs on complex geometrical domains, how to efficiently apply the IIF temporal discretization was open. In this paper, we solve this problem by applying the Krylov subspace approximations to the matrix exponential operator. Then we apply this novel time discretization technique to discontinuous Galerkin (DG) methods on unstructured meshes for solving reaction-diffusion equations. Numerical examples are shown to demonstrate the accuracy, efficiency and robustness of the method in resolving the stiffness of the DG spatial operator for reaction-diffusion PDEs. Application of the method to a mathematical model in pattern formation during zebrafish embryo development shall be shown.
\end{abstract}

(c) 2011 Elsevier Inc. All rights reserved.

\section{Introduction}

High order accuracy numerical methods (e.g. weighted essentially non-oscillatory (WENO) methods, discontinuous Galerkin (DG) methods, spectral methods, etc.) are especially efficient for numerically solving problems which contain complex solution structures, and have been applied extensively in computational fluid dynamics. Efficient and high order temporal numerical schemes are important for the performance of high accuracy numerical simulations. A lot of state-of-the-art high order time-stepping methods were developed. Here we just give a few examples and it is not a complete list. For example, the total variation diminishing (TVD) Runge-Kutta (RK) schemes [54,55,28,29]; high order implicit-explicit (IMEX) multistep/Runge-Kutta methods and their applications [2,3,34,36,47,59,51,68]; spectral deferred correction (SDC) methods $[9,24,33,41,42,45]$; hybrid methods of SDC and high order RK schemes [12]; etc.

\footnotetext{
* Corresponding author. Tel.: +1 5746316079 .

E-mail addresses: chen39@iusb.edu (S. Chen), yzhang10@nd.edu (Y.-T. Zhang).

${ }^{1}$ Research supported by IU South Bend Research and Development grant.

${ }^{2}$ Research partially supported by NSF grant DMS-0810413 and Oak Ridge Associated Universities (ORAU) Ralph E. Powe Junior Faculty Enhancement Award.
} 
Integration factor (IF) methods are a broad class of time discretization methods which have been combined with spatial discretization methods especially spectral methods $[25,8,10,56]$ for solving various partial differential equations (PDEs). They are especially useful for many important mathematical models in fluid dynamics or biological problems which involve nonlinear PDEs with the linear highest spatial derivatives. The method of lines generates stiff systems of ordinary differential equations (ODEs) with the stiff linear terms (nonlinear terms could also be stiff, depending on different problems). Integration factor methods are a class of "exactly linear part" time discretization methods for the solution of this type of stiff systems. This class of methods perform the time evolution of the stiff linear operator via evaluation of an exponential function of the corresponding matrix. Hence for PDEs with linear high order derivatives, the integration factor type time discretization can remove both the stability constrain and numerical errors from the high order derivatives.

Traditional integration factor methods have a disadvantage that large error coefficients are produced when the linear term has a large norm [8]. Recently Krogstad developed a method which can improve the accuracy of the traditional integration factor methods significantly [38]. Another class of "integration factor" type methods, the exponential time differencing (ETD) methods [5,6,22,35], can also obtain a better accuracy than the traditional integration factor methods. For ETD methods, extra treatments are needed for consistent order of accuracy [35,23].

The explicit integration factor methods use explicit linear multi-step methods or explicit Runge-Kutta methods to treat the nonlinear terms in the system (e.g. the explicit ETD-RK schemes [22]), which are very efficient for systems with stiff linear part but nonstiff or mildly stiff nonlinear part. In [32], explicit exponential Runge-Kutta methods for the time integration of semilinear parabolic problems were analyzed. For systems with both stiff linear and nonlinear terms, an implicit treatment of nonlinear terms is desirable. In [48], we developed a class of efficient implicit integration factor (IIF) methods for solving systems with both stiff linear and nonlinear terms. A novel property of the methods is that the implicit terms are free of the exponential operation of the linear terms. Hence when the methods are applied to PDEs with stiff nonlinear reactions (e.g. the reaction-diffusion systems arising from mathematical models in computational developmental biology), the exact evaluation of the linear part is decoupled from the implicit treatment of the nonlinear reaction terms. As a result, the size of the nonlinear system arising from the implicit treatment is independent of the number of spatial grid points; it only depends on the number of the original PDEs. This distinguishes our IIF methods [48] from implicit ETD methods in [6]. Another efficient approach to decouple the stiff nonlinear terms from the linear terms is to use operator splitting integration factor methods (e.g. [44]). We would like to point out that our approach is different from the operator splitting methods, hence is free of operator splitting errors.

The tremendous difficulty in implementing integration factor type method for high dimensional problems is how to efficiently evaluate the product of the matrix exponential and a vector. The differential matrix from the high dimensional spatial discretization is usually very large and sparse, but its exponential matrix will be dense. CPU cost and storage of such exponential matrix are prohibitive for high dimensional problems, although it can be handled well for 1D problems [48]. On rectangular meshes, the compact integration factor methods [49] can be used to deal with this issue. By introducing a compact representation for the matrix approximating the differential operator, the compact IIF methods apply matrix exponential operations sequentially in every spatial direction. As the results, exponential matrices which are calculated and stored have small sizes, as those in the 1D problem. For example for a 3D problem, if we have $N$ grid points in every direction of the $x, y$ and $z$ directions, then we do not need to work on the full $N^{3} \times N^{3}$ matrix and its exponential as in the original non-compact IIF methods [48], but just need to consider several $N \times N$ matrices and their exponentials. The storage and CPU time required for compact IIF schemes are smaller by orders of magnitude than the non-compact ones. The operation count of compact integration factor schemes is $O\left(N^{4}\right)$ vs. $O\left(N^{6}\right)$ for non-compact ones. See more details in [49]. But how to apply this approach to spatial discretization on high dimensional unstructured meshes for dealing with complex domain geometry is still unclear. In this paper, we address this problem by using the Krylov subspace approximations to the matrix exponential operator and develop the Krylov implicit integration factor (Krylov IIF) methods for spatial discretization on triangular meshes.

The Krylov subspace methods were used and analyzed by Gallopoulos and Saad [26], Saad [52] for the approximation of a product of a exponential matrix of a large sparse differential matrix and a given vector, in the finite difference discretization of a time-dependent diffusion equation. Since in many applications including the integration factor methods, one does not need the full exponential matrix, but only the matrix-vector product. See the review paper [46]. As discovered in [31], the convergence to $e^{A \Delta t} v$ is substantially faster than that of corresponding Krylov methods for the solution of linear system $(I-\Delta t A) x=v$, which arises in the implicit treatment of diffusion or other high order derivative terms, at least unless a good preconditioner is available.

For the spatial directions, discontinuous Galerkin (DG) finite element methods are a class of popular high order accuracy spatial discretization methods for numerically solving various partial differential equations (PDEs) arising from computational fluid dynamics and other computational physics problems. DG methods confer several advantages that make them attractive for applications. These include common advantages shared by all finite element methods such as their ability for easy handling of complicated geometry and boundary conditions. Since DG methods use completely discontinuous piecewise polynomial space for the numerical solution and the test functions, this property makes DG methods have lots of flexibility which is not shared by continuous Galerkin finite element methods, such as their flexibility for easy $h-p$ adaptivity including changes of approximation orders between neighboring elements and allowing general meshes with hanging nodes, their compactness hence efficient parallel implementation [7], and their easy coordination with finite volume techniques for computing problems with discontinuous or sharp gradient solutions. The first DG method was introduced by Reed and Hill [50], in the framework of neutron transport. A major development of DG methods was carried out by Cockburn et al. in a 
series of papers $[16,17,14,18,19]$, in which they established a framework to easily solve nonlinear time dependent hyperbolic conservation laws. Good references for DG methods and their recent development can be found in the review papers $[15,13,20]$.

In recent years, DG methods have been generalized to solve time dependent PDEs containing higher spatial derivatives. This class of new methods are termed local discontinuous Galerkin (LDG) methods. See e.g. [4,21,43,61-66,37]. In a recent paper [11], Cheng and Shu developed a new DG method for solving time dependent PDEs with higher order spatial derivatives, based on $[1,27,58]$. The scheme is formulated by repeated integration by parts of the original equation and then replacing the interface values of the solution by carefully chosen numerical fluxes. When DG methods are applied to discretize the spatial variables for convection-diffusion problems or other PDEs which have high order derivatives, a large coupled stiff ordinary differential equations (ODEs) system is generated. An explicit time discretization for the resulting stiff ODE system will suffer from extremely small time step restriction for stability. In [60], time discretization techniques including semi-implicit spectral deferred correction method, the additive Runge-Kutta method and the exponential time differencing (ETD) method for solving the stiff ODEs resulting from a LDG spatial discretization to PDEs with high order spatial derivatives on $1 \mathrm{D}$ spatial domain were compared and studied.

To test our new Krylov IIF schemes in this paper, we apply them to solve the stiff ODE system resulting from a DG spatial discretization for reaction-diffusion problems on 2D triangular meshes [69]. Due to the stiffness of the spatial operators, the implicit discretization was formulated for the $P^{1}$ case in [69] and an operator-splitting approach was used to enhance the computation efficiency. The DG spatial discretization in [69] is based on [11], but the numerical fluxes are different from those in [11]. In this paper, we will formulate the implicit DG discretization for both the $P^{1}$ and $P^{2}$ cases, and apply the new Krylov IIF schemes for the time-marching. Numerical examples are shown to demonstrate the accuracy, efficiency and robustness of the method in resolving the stiffness of the DG spatial operator for reaction-diffusion PDEs which have higher than first order spatial derivatives. Application of the method to a mathematical model in spatial pattern formation during zebrafish embryo development shall be shown.

The rest of the paper is organized as following. In Section 2, we derive and formulate the Krylov IIF methods. In Section 3, we describe in detail the $P^{1}$ and $P^{2}$ DG spatial discretization for reaction-diffusion equations on 2D triangular meshes; then apply the new Krylov IIF methods to the resulting stiff ODE systems. Numerical experiments are presented in Section 4. Discussions and conclusions are given in Section 5.

\section{Implicit integration factor methods based on Krylov subspace approximation}

We first review the original IIF methods developed in [48]. Then we propose the new IIF methods based on Krylov subspace approximations to the matrix exponential operator.

\subsection{Review of the original IIF methods}

Assume that we need to solve a system of ODEs which arises after a spatial discretization of a PDE system whose highest order term is linear and has higher than first order derivative (e.g. a reaction-diffusion equation):

$$
\frac{d U(t)}{d t}=\mathbf{A} U(t)+\mathbf{F}(U(t))
$$

where $U(t) \in R^{N}, \mathbf{A} \in R^{N \times N}$ is the constant approximation matrix for the linear differential operator of the highest order derivative, $\mathbf{F}(U(t)) \in R^{N}$ is the nonlinear term. The matrix $\mathbf{A}$ is usually a sparse matrix when a finite difference or finite element method is used for the spatial discretization.

To construct the IIF methods for (1), we multiply it by the integration factor $e^{-\mathbf{A} t}$ and integrate over one time step from $t_{n}$ to $t_{n+1} \equiv t_{n}+\Delta t$ to obtain

$$
U\left(t_{n+1}\right)=e^{\mathbf{A} \Delta t} U\left(t_{n}\right)+e^{\mathbf{A} \Delta t} \int_{0}^{\Delta t} e^{-\mathbf{A} \tau} \mathbf{F}\left(U\left(t_{n}+\tau\right)\right) d \tau .
$$

Then we approximate the integrand in (2) by using an $r$ - 1th order Lagrange interpolation polynomial with interpolation points at $t_{n+1}, t_{n}, \ldots, t_{n+2-r}$, and obtain the $r$ th order IIF scheme

$$
U_{n+1}=e^{\mathbf{A} \Delta t} U_{n}+\Delta t\left(\alpha_{1} \mathbf{F}\left(U_{n+1}\right)+\sum_{i=0}^{r-2} \alpha_{-i} e^{(i+1) \mathbf{A} \Delta t} \mathbf{F}\left(U_{n-i}\right)\right)
$$

where

$$
\alpha_{-i}=\frac{1}{\Delta t} \int_{0}^{\Delta t} \prod_{\substack{k=-1 \\ k \neq i}}^{r-2} \frac{\tau+k \Delta t}{(k-i) \Delta t} d \tau, \quad-1 \leqslant i \leqslant r-2 .
$$

See $[48,49]$ for the values of coefficients $\alpha_{j}$ for the schemes with different orders. For example, the second order scheme is of the following form 


$$
U_{n+1}=e^{\mathbf{A} \Delta t}\left(U_{n}+\frac{\Delta t}{2} \mathbf{F}\left(U_{n}\right)\right)+\frac{\Delta t}{2} \mathbf{F}\left(U_{n+1}\right)
$$

and the third order scheme is

$$
U_{n+1}=e^{\mathbf{A} \Delta t} U_{n}+\Delta t\left(\frac{5}{12} \mathbf{F}\left(U_{n+1}\right)+\frac{2}{3} e^{\mathbf{A} \Delta t} \mathbf{F}\left(U_{n}\right)-\frac{1}{12} e^{2 \mathbf{A} \Delta t} \mathbf{F}\left(U_{n-1}\right)\right) .
$$

The distinct feature of the scheme (3) is that the nonlinear implicit term $\mathbf{F}\left(U_{n+1}\right)$ does not involve the matrix exponential operator, unlike the implicit ETD schemes [6]. Hence if the nonlinear operator $\mathbf{F}$ itself does not involve the coupling of the numerical values at the spatial grid points (e.g. the nonlinear reactions), the size of nonlinear system resulted from the implicit treatment is independent of the number of the spatial grid points, and it only depends on the number of the original PDEs.

Remark. We consider the time step size $\Delta t$ to be uniform in the time evolution in this paper. The methods to efficiently deal with non-uniform time step sizes (e.g., in adaptive time step computation; in solving PDEs with hyperbolic terms and the CFL condition constraint) will be reported in the future.

\subsection{IIF methods based on Krylov subspace approximation}

The efficiency of the IIF schemes ( 3 ) largely depends on the methods to evaluate the product of the matrix exponential and a vector, for example $e^{\mathbf{A} \Delta t} U_{n}$. For PDEs defined on high spatial dimensions (two spatial dimensions (2D) and above), the method of lines (MOL) with a specific spatial discretization will generate a large and sparse matrix $\mathbf{A}$ in (1). But the exponential matrix $e^{\mathbf{A} \Delta t}$ is dense. For example if a finite difference method is used on a 2D rectangular mesh with $N$ grid points in both $x$ and $y$ directions, then the matrix $\mathbf{A}$ has size $N^{2} \times N^{2}$. In [48], for a given spatial and temporal numerical resolution, the exponential matrices such as $e^{\mathbf{A} \Delta t}$ are pre-computed and stored for later use at every time step. For one-dimensional problems, this works very well since the size of the matrix $\mathbf{A}$ is manageable. For 2D and 3D problems, direct computation and storage of such exponential matrix are prohibitive in terms of both CPU cost and computer memory.

On rectangular meshes, the compact IIF methods [49] can be used to deal with this issue for some differential operators (e.g. the Laplacian operator). The compact IIF methods introduce a compact representation for the matrix approximating the differential operator. The compact form involves storage only proportional to the number of unknowns, i.e. the size of $U$, unlike the non-compact approach, which is proportional to the square of the unknowns. As a result, exponential matrices which are calculated and stored have small sizes, as those in the 1D problem. For example, for a 3D problem if we have $N_{x}, N_{y}$ and $N_{z}$ grid points in $x, y$ and $z$ directions, then instead of considering the full $\left(N_{x} N_{y} N_{z}\right) \times\left(N_{x} N_{y} N_{z}\right)$ matrix and its exponential, we just consider $N_{x} \times N_{x}, N_{y} \times N_{y}$ and $N_{z} \times N_{z}$ matrices and their exponentials. The storage and CPU time required for compact IIF schemes are smaller by orders of magnitude than the original non-compact ones in [48]. The operation count of compact IIF schemes is $O\left(N_{x}^{2} N_{y} N_{z}+N_{x} N_{y}^{2} N_{z}+N_{x} N_{y} N_{x}^{2}\right)$ vs. $O\left(N_{x}^{2} N_{y}^{2} N_{z}^{2}\right)$ for non-compact IIF schemes.

For spatial discretization on high dimensional unstructured meshes (e.g. triangular meshes), how to formulate a compact matrix representation of the spatial operations and how to apply this approach are still unclear. Moreover, application of compact IIF methods to more general spatial operators (e.g. the general diffusion operators involving mixed derivatives) is not straightforward. So we re-consider the non-compact IIF methods (3) and look for another way to efficiently compute the matrix exponential operations.

Although the matrix $\mathbf{A}$ is sparse for many spatial discretizations, the exponential matrix $e^{\mathbf{A} \Delta t}$ is dense. Directly computing and storing $e^{\mathbf{A} \Delta t}$ for spatial discretization on high dimensional unstructured meshes are not practical for a typical machine. Fortunately we do not need the full exponential matrix $e^{\mathbf{A} \Delta t}$ itself, but only the products of the exponential matrix and some vectors in (3). The Krylov subspace approximations to the matrix exponential operator is an excellent choice in terms of both accuracy and efficiency. For example, in [26,52], the Krylov subspace methods were used for the approximation of $e^{\mathbf{A} \Delta t} v$, where $\mathbf{A}$ is a large sparse matrix and $v$ is a given vector, in the finite difference discretization of a time-dependent diffusion equation. Next we apply the Krylov subspace method to approximate the products of the exponential matrix and vectors in our IIF schemes ( 3 ) and derive the new Krylov IIF methods. First we describe the Krylov subspace methods to approximate $e^{\mathbf{A} \Delta t} v$, following the literature (e.g. $\left.[26,46]\right)$.

The large sparse matrix $\mathbf{A}$ is projected to the Krylov subspace

$$
K_{M}=\operatorname{span}\left\{v, \mathbf{A} v, \mathbf{A}^{2} v, \ldots, \mathbf{A}^{M-1} v\right\} .
$$

The dimension $M$ of the Krylov subspace is much smaller than the dimension $N$ of the large sparse matrix $\mathbf{A}$. In all numerical computations of this paper, we take $M=25$ for different $N$, and accurate results are obtained as shown in Section 4 . An orthonormal basis $V_{M}=\left[v_{1}, v_{2}, v_{3}, \ldots, v_{M}\right]$ of the Krylov subspace $K_{M}$ is generated by the well-known Arnoldi algorithm [57] as the following.

1. Compute the initial vector: $v_{1}=v /\|v\|_{2}$. 
2. Perform iterations: Do $j=1,2, \ldots, M$ :

(1) Compute the vector $w=\mathbf{A} v_{j}$.

(2) Do $i=1,2, \ldots, j$ :

(a) Compute the inner product $h_{i, j}=\left(w, v_{i}\right)$.

(b) Compute the vector $w=w-h_{i, j} v_{i}$.

(3) Compute $h_{j+1, j}=\|w\|_{2}$.

(4) If $h_{j+1, j} \equiv 0$, then stop the iteration; else compute the next basis vector $v_{j+1}=w / h_{j+1 j}$.

In the Arnoldi algorithm, if $h_{j+1, j} \equiv 0$ for some $j<M$, it means that the convergence has occurred and the Krylov subspace $K_{M}=\operatorname{span}\left\{v_{1}, v_{2}, \ldots, v_{j}\right\}$, so the iteration can be stopped at this step $j$, and we assign the value of this $j$ to $M$. This algorithm will produce an orthonormal basis $V_{M}$ of the Krylov subspace $K_{M}$. Denote the $M \times M$ upper Hessenberg matrix consisting of the coefficients $h_{i, j}$ by $H_{M}$. Since the columns of $V_{M}$ are orthogonal, we have

$$
H_{M}=V_{M}^{T} \mathbf{A} V_{M}
$$

This means that the very small Hessenberg matrix $H_{M}$ represents the projection of the large sparse matrix A to the Krylov subspace $K_{M}$, with respect to the basis $V_{M}$. Also since $V_{M}$ is orthonormal, the vector $V_{M} V_{M}^{T} e^{\mathbf{A} \Delta t} v$ is the orthogonal projection of $e^{\mathbf{A} \Delta t} v$ on the Krylov subspace $K_{M}$, namely, it is the closest approximation to $e^{\mathbf{A} \Delta t} v$ from $K_{M}$. Therefore

$$
e^{\mathbf{A} \Delta t} v \simeq V_{M} V_{M}^{T} e^{\mathbf{A} \Delta t} v=\beta V_{M} V_{M}^{T} e^{\mathbf{A} \Delta t} v_{1}=\beta V_{M} V_{M}^{T} e^{\mathbf{A} \Delta t} V_{M} e_{1}
$$

where $\beta=\|v\|_{2}$, and $e_{1}$ denotes the first column of the $M \times M$ identity matrix $I_{M}$. Use the fact of (8), we have the approximation

$$
e^{\mathbf{A} \Delta t} v \simeq \beta V_{M} e^{H_{M} \Delta t} e_{1}
$$

Thus the large $e^{\mathbf{A} \Delta t}$ matrix exponential problem is replaced with a much smaller $e^{H_{M} \Delta t}$ problem. The small matrix exponential $e^{H_{M} \Delta t}$ will be computed using a scaling and squaring algorithm with a Padé approximation with only computational cost of $O\left(M^{2}\right)$, see $[30,46,26]$. Applying the Krylov subspace approximation (9) to (3), we obtain the Krylov IIF schemes

$$
U_{n+1}=\Delta t \alpha_{1} \mathbf{F}\left(U_{n+1}\right)+\beta_{0, n} V_{M, 0, n} e^{H_{M, 0, n} \Delta t} e_{1}+\Delta t\left(\sum_{i=1}^{r-2} \alpha_{-i} \beta_{i, n} V_{M, i, n} e^{(i+1) H_{M, i, n} \Delta t} e_{1}\right)
$$

where $\beta_{0, n}=\left\|U_{n}+\alpha_{0} \Delta t \mathbf{F}\left(U_{n}\right)\right\|_{2}, \beta_{i, n}=\left\|\mathbf{F}\left(U_{n-i}\right)\right\|_{2}, V_{M, 0, n}$ and $H_{M, 0, n}$ are orthonormal basis and upper Hessenberg matrix generated by the Arnoldi algorithm with the initial vector $U_{n}+\alpha_{0} \Delta t \mathbf{F}\left(U_{n}\right) . V_{M, i, n}$ and $H_{M, i, n}$ are orthonormal basis and upper Hessenberg matrix generated by the Arnoldi algorithm with the initial vectors $\mathbf{F}\left(U_{n-i}\right)$, for $i=1,2, \ldots, r-2$. We would like to point out that $V_{M, 0, n}$ and $V_{M, i, n}, i=1,2, \ldots, r-2$ are orthonormal bases of different Krylov subspaces for the same matrix A, which are generated with different initial vectors in the Arnoldi algorithm. The value of $M$ is taken to be large enough such that the errors of Krylov subspace approximations are much less than the truncation errors of the numerical schemes (3). From our numerical experiments in this paper (Section 4), we can see that our numerical schemes have already given a clear accuracy order with a very small size $M=25$, and $M$ does not need to be increased when the spatial-temporal resolution is refined. Specifically the second order Krylov IIF scheme has the form

$$
U_{n+1}=\frac{1}{2} \Delta t \mathbf{F}\left(U_{n+1}\right)+\beta_{n} V_{M, n} e^{H_{M, n} \Delta t} e_{1},
$$

where $\beta_{n}=\left\|U_{n}+\frac{1}{2} \Delta t \mathbf{F}\left(U_{n}\right)\right\|_{2}, V_{M, n}$ and $H_{M, n}$ are orthonormal basis and upper Hessenberg matrix generated by the Arnoldi algorithm with the initial vector $U_{n}+\frac{1}{2} \Delta t \mathbf{F}\left(U_{n}\right)$. And the third order Krylov IIF scheme has the form

$$
U_{n+1}=\frac{5}{12} \Delta t \mathbf{F}\left(U_{n+1}\right)+\beta_{0, n} V_{M, 0, n} e^{H_{M, 0, n} \Delta t} e_{1}-\frac{1}{12} \Delta t \beta_{1, n} V_{M, 1, n} e^{2 H_{M, 1, n} \Delta t} e_{1},
$$

where $\beta_{0, n}=\left\|U_{n}+\frac{2}{3} \Delta t \mathbf{F}\left(U_{n}\right)\right\|_{2}, \beta_{1, n}=\left\|\mathbf{F}\left(U_{n-1}\right)\right\|_{2}, V_{M, 0, n}$ and $H_{M, 0, n}$ are orthonormal basis and upper Hessenberg matrix generated by the Arnoldi algorithm with the initial vector $U_{n}+\frac{2}{3} \Delta t \mathbf{F}\left(U_{n}\right) . V_{M, 1, n}$ and $H_{M, 1, n}$ are orthonormal basis and upper Hessenberg matrix generated by the Arnoldi algorithm with the initial vector $\mathbf{F}\left(U_{n-1}\right)$.

Remark. we would like to emphasize that the Krylov IIF schemes given in (10)-(12) are novel methods which combine the original IIF schemes with the Krylov subspace approximations. These new methods are designed to deal with more general and complex problems. 


\section{Implicit DG formulations and application of Krylov IIF methods}

To test our Krylov IIF methods, we apply them to solve the stiff ODE system arising from a DG discretization of reactiondiffusion equations on 2D triangular meshes. Let $\Omega$ be an open, bounded domain on which the reaction-diffusion system

$$
\frac{\partial \mathbf{u}}{\partial t}=D \Delta \mathbf{u}+F(\mathbf{u})
$$

is defined, where $\mathbf{u}$ often represents concentrations of a group of chemical molecules, $D$ is the constant diffusion matrix, $\Delta \mathbf{u}$ is the Laplacian associated with the diffusion of the molecules $\mathbf{u}$, and $F(\mathbf{u})$ describes the nonlinear chemical reactions. For the simplicity of the description, we consider the scalar case of (13). The numerical formulae for the scalar case can be straightforwardly extended to solve the system case component by component. We consider a triangulation $\Omega_{h}$ of $\Omega$ which consists of non-overlapping triangles $\left\{\Delta_{m}\right\}_{m=1}^{N}$. Let $h_{\min }=\min _{1 \leqslant m \leqslant N} \rho_{m}$, where $\rho_{m}$ is the diameter of the inscribed circle of the triangle $\Delta_{m}$.

Define the finite element space $V_{h}^{k}=\left\{v:\left.v\right|_{\Delta m} \in P^{k}\left(\Delta_{m}\right), m=1, \ldots, N\right\}$, where $P^{k}\left(\Delta_{m}\right)$ denotes the set of all polynomials of degree at most $k$ on $\triangle_{m}$.

We apply the DG formulation in [69] to discretize the reaction-diffusion Eq. (13) in the spatial direction, and keep the time variable continuous. This DG discretization is based on a DG formulation for convection-diffusion equations in [11], but we use different numerical fluxes for the diffusion term. The semi-discrete scheme is: find $u \in V_{h}^{k}$, such that

$$
\int_{\Delta_{m}} u_{t} v d x-D \int_{\triangle_{m}} u \nabla^{2} v d x+D \int_{\partial \triangle_{m}} \hat{u} \nabla v \cdot \vec{n}_{\partial \triangle_{m}} d S-D \int_{\partial \triangle_{m}} v \widetilde{\nabla u} \cdot \vec{n}_{\partial \triangle_{m}} d S=\int_{\triangle_{m}} F(u) v d x
$$

holds true for any $v \in V_{h}^{k}$ and $m=1, \ldots, N$. The numerical fluxes on the element edges $\partial \Delta_{m}$ are chosen as

$$
\begin{aligned}
& \hat{u}=\frac{u^{i n}+u^{e x t}}{2}, \\
& \widetilde{\nabla u}=\frac{(\nabla u)^{i n}+(\nabla u)^{e x t}}{2}+\beta[u],
\end{aligned}
$$

where the jump term

$$
[u]=\left.\left(u^{e x t}-u^{i n}\right)\right|_{\partial \triangle_{m}} \cdot \vec{n}_{\partial \Delta_{m}},
$$

$u^{i n}$ and $u^{e x t}$ are the limits of $u$ at $x \in \partial \triangle_{m}$ taken from the interior and the exterior of $\triangle_{m}$, respectively, $\vec{n}_{\partial \Delta_{m}}$ is the outward unit normal to the element $\Delta_{m}$ at $x \in \partial \triangle_{m}$, and $\beta$ is a positive constant that is of the order $\mathcal{O}\left(h_{\min }^{-1}\right)$. In all computations in this paper, we take $\beta=10 / h_{\min }$. The choice of numerical fluxes (15)-(17) is crucial for the stability and convergence of the DG scheme (14). We use the central/ average numerical fluxes for the diffusion terms. In [11], the alternative numerical fluxes were used for the diffusion terms. The differences of the central and alternative numerical fluxes were discussed in [20] for the LDG method. We will explore the application of the alternative numerical fluxes [11] to the DG schemes (14) on triangular meshes in our future work.

To apply the Krylov IIF schemes (10) to the DG spatial discretization (14), we will need the matrix expression (i.e., the implicit DG formulae) as (1). This is a different step from the explicit DG methods for hyperbolic conservation laws. The implicit DG formulae for the $P^{1}$ case has been given in [69]. In this paper, we will present the implicit DG formulae for both the $P^{1}$ and $P^{2}$ cases. For each element $\Delta_{m}$, we denote its three neighboring elements by $i_{m}, j_{m}$, and $k_{m}$. To simplify notations in the following presentation, we will omit the subscript $m$ and just use $i, j, k$ to represent the neighboring cells of $\Delta_{m}$.

The implicit DG formulae for the $P^{1}$ case has been derived in [69]. But to make the paper self-contained, we will re-present the $P^{1}$ case in Section 3.1. The new formulae for the $P^{2}$ case will be given in Section 3.2.

\subsection{The $P^{1}$ case}

The linear polynomial on $\Delta_{m}$ is represented by

$$
u(x, y, t)=a_{m}(t)+b_{m}(t) \xi_{m}+c_{m}(t) \eta_{m},
$$

where

$$
\begin{aligned}
& \xi_{m}=\frac{x-x_{m}}{h_{m}}, \\
& \eta_{m}=\frac{y-y_{m}}{h_{m}}
\end{aligned}
$$

and $\left(x_{m}, y_{m}\right)$ is the barycenter of the element $\Delta_{m}, h_{m}=\sqrt{\left|\Delta_{m}\right|}$ with $\left|\Delta_{m}\right|$ denoting the area of the triangle $\Delta_{m}$. By taking $v=1$, $\xi_{m}, \eta_{m}$ on $\Delta_{m}$ and $v=0$ elsewhere, the DG formulation (14) can be converted from the integral form to the following semidiscretized ODE system, for $m=1, \ldots, N$ :

$$
q_{11} a_{m}^{\prime}(t)+q_{12} b_{m}^{\prime}(t)+q_{13} c_{m}^{\prime}(t)=D \sum_{l=m, i, j, k}\left[w_{a l 1} a_{l}(t)+w_{b l 1} b_{l}(t)+w_{c l 1} c_{l}(t)\right]+\left(q_{11} / 3\right) \sum_{l=i, j, k} F\left(u\left(x_{m, l}, y_{m, l}\right)\right),
$$




$$
\begin{aligned}
& q_{21} a_{m}^{\prime}(t)+q_{22} b_{m}^{\prime}(t)+q_{23} c_{m}^{\prime}(t)=D \sum_{l=m, i, j, k}\left[w_{a l 2} a_{l}(t)+w_{b l 2} b_{l}(t)+w_{c l 2} c_{l}(t)\right]+\left(q_{11} / 3\right) \sum_{l=i, j, k} F\left(u\left(x_{m, l}, y_{m, l}\right)\right) \xi_{m}\left(x_{m, l}, y_{m, l}\right), \\
& q_{31} a_{m}^{\prime}(t)+q_{32} b_{m}^{\prime}(t)+q_{33} c_{m}^{\prime}(t)=D \sum_{l=m, i, j, k}\left[w_{a l 3} a_{l}(t)+w_{b l 3} b_{l}(t)+w_{c l 3} c_{l}(t)\right]+\left(q_{11} / 3\right) \sum_{l=i, j, k} F\left(u\left(x_{m, l}, y_{m, l}\right)\right) \eta_{m}\left(x_{m, l}, y_{m, l}\right),
\end{aligned}
$$

where the coefficients $\left\{q_{r s}\right\}_{r, s=1}^{3},\left\{\left\{w_{a l r}\right\}_{r=1}^{3},\left\{w_{b l r}\right\}_{r=1}^{3},\left\{w_{c l r}\right\}_{r=1}^{3}\right\}_{l=m, i, j, k}$ are constants which depend on the local geometry of the mesh (i.e., triangle $\Delta_{m}$ and its neighboring cells $i, j, k$ and $\vec{n}_{\partial \Delta_{m}}$ ), the local basis functions $1,\left\{\xi_{l}, \eta_{l}\right\}_{l=m, i, j, k}$, and the constant $\beta$. $\left\{\left(x_{m, l}, y_{m, l}\right)\right\}_{l=i, j, k}$ are the mid-points of the three edges $\left\{e_{l}\right\}_{l=i, j, k}$ of $\triangle_{m}$ which serve as Gaussian quadrature points for the $P^{1}$ case in the integral involving the nonlinear reaction terms in (14). The detailed formulae for computing these constants are presented in the technical report which is available at "http://www.nd.edu/ yzhang10/IIF-DG-report.pdf". In our implementation, these mesh-dependent constants are pre-calculated and stored before the time evolution since they do not depend on the numerical solution $u$. Rewrite Eqs. (21)-(23) to the matrix-vector form

$\begin{aligned} \mathbf{Q}_{m} \vec{V}_{m}^{\prime}(t) & =D \sum_{l=m, i, j, k} \mathbf{W}_{l} \underset{l}{\vec{V}}(t)+\underset{m}{\vec{F}}(\underset{m}{\vec{V}}) \quad m=1, \ldots, N, \\ \text { where } \quad \mathbf{Q}_{m} & =\left(\begin{array}{lll}q_{11} & q_{12} & q_{13} \\ q_{21} & q_{22} & q_{23} \\ q_{31} & q_{32} & q_{33}\end{array}\right), \mathbf{W}_{l}=\left(\begin{array}{lll}w_{a l 1} & w_{b l 1} & w_{c l 1} \\ w_{a l 2} & w_{b l 2} & w_{c l 2} \\ w_{a l 3} & w_{b l 3} & w_{c l 3}\end{array}\right), \vec{V}_{m}=\left(\begin{array}{l}a_{m}(t) \\ b_{m}(t) \\ c_{m}(t)\end{array}\right), \vec{V}_{l}=\left(\begin{array}{l}a_{l}(t) \\ b_{l}(t) \\ c_{l}(t)\end{array}\right), \quad \text { and } \quad \vec{F}_{m}\left(\vec{V}_{m}\right)=\end{aligned}$ $\left(\begin{array}{c}\left(q_{11} / 3\right) \sum_{l=i, j, k} F\left(u\left(x_{m, l}, y_{m, l}\right)\right) \\ \left(q_{11} / 3\right) \sum_{l=i, j, k} F\left(u\left(x_{m, l}, y_{m, l}\right)\right) \xi_{m}\left(x_{m, l}, y_{m, l}\right) \\ \left(q_{11} / 3\right) \sum_{l=i, j, k} F\left(u\left(x_{m, l}, y_{m, l}\right)\right) \eta_{m}\left(x_{m, l}, y_{m, l}\right)\end{array}\right)$.

\subsection{The $P^{2}$ case}

The procedure to construct the $P^{2}$ implicit DG spatial discretizations with third order accuracy will follow that of the $P^{1}$ case, with a larger semi-discretized system. The number of local equations on each triangle depends on the degree of freedoms of the approximation polynomial. On each triangle $\Delta_{m}$, a quadratic polynomial

$$
u(x, y, t)=a_{m}(t)+b_{m}(t) \xi_{m}+c_{m}(t) \eta_{m}+d_{m}(t) \xi_{m} \eta_{m}+e_{m}(t) \xi_{m}^{2}+f_{m}(t) \eta_{m}^{2}
$$

will be constructed. In (14), we take $v=1, \xi_{m}, \eta_{m}, \xi_{m} \eta_{m}, \xi_{m}^{2}, \eta_{m}^{2}$ on $\triangle_{m}$ and $v=0$ elsewhere and convert the integral formulation to the semi-discretized ODE system

$$
\mathbf{Q}_{m} \vec{V}_{m}^{\prime}(t)=D \sum_{l=m, i, j, k} \mathbf{W}_{l} \vec{V}_{l}(t)+\vec{F}_{m}\left(\vec{V}_{m}\right) \quad m=1, \ldots, N
$$

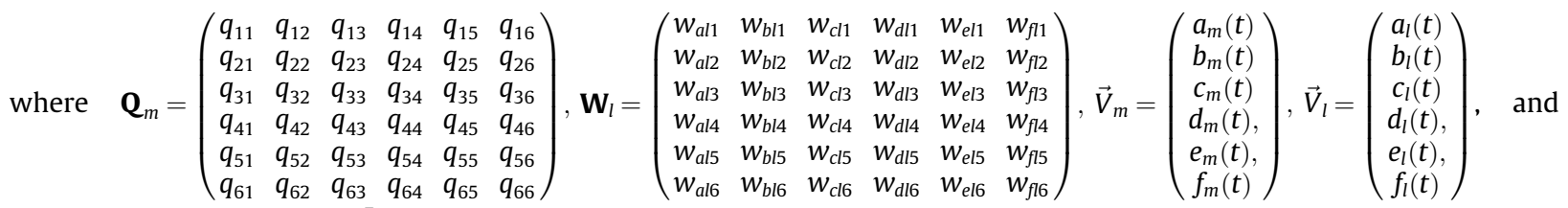
$\vec{F}_{m}\left(\vec{V}_{m}\right)=\left(\begin{array}{c}q_{11} \sum_{v=1}^{7} \omega_{v} F\left(u\left(x_{m_{v}}, y_{m_{v}}\right)\right) \\ q_{11} \sum_{v=1}^{7} \omega_{v} F\left(u\left(x_{m_{v}}, y_{m_{v}}\right)\right) \xi_{m}\left(x_{m_{v}}, y_{m_{v}}\right) \\ q_{11} \sum_{v=1}^{7} \omega_{v} F\left(u\left(x_{m_{v}}, y_{m_{v}}\right)\right) \eta_{m}\left(x_{m_{v}}, y_{m_{v}}\right) \\ q_{11} \sum_{v=1}^{7} \omega_{v} F\left(u\left(x_{m_{v}}, y_{m_{v}}\right)\right) \xi_{m}\left(x_{m_{v}}, y_{m_{v}}\right) \eta_{m}\left(x_{m_{v}}, y_{m_{v}}\right) \\ q_{11} \sum_{v=1}^{7} \omega_{v} F\left(u\left(x_{m_{v}}, y_{m_{v}}\right)\right) \xi_{m}^{2}\left(x_{m_{v}}, y_{m_{v}}\right) \\ q_{11} \sum_{v=1}^{7} \omega_{v} F\left(u\left(x_{m_{v}}, y_{m_{v}}\right)\right) \eta_{m}^{2}\left(x_{m_{v}}, y_{m_{v}}\right)\end{array}\right)$.

Again the detailed formulae for elements of matrices $\mathbf{Q}_{m}, \mathbf{W}_{m}$ and $\mathbf{W}_{l}, l=i, j, k$ are given in the technical report which is available at "http://www.nd.edu/ yzhang10/IIF-DG-report.pdf". These mesh-dependent constants do not depend on the numerical solution $u$ and are pre-calculated and stored before the time evolution. $\left(x_{m_{v}}, y_{m_{v}}\right), v=1, \ldots, 7$ are the Gaussian quadrature points in the triangle $\Delta_{m}$ for computing the integral involving the nonlinear reaction terms for the $P^{2}$ case. Their values are also given in the aforementioned technical report.

Remark. The procedure to construct the $P^{3}$ or higher order implicit DG spatial discretization will follow the similar procedure as for the $P^{1}$ and $P^{2}$ cases. The local ODE systems will have the same form as (24) and (26), but with larger sizes which depend on the degrees of freedom of the approximation polynomials. For example, if the degree of freedom of the approximation polynomial is $p$ on each element, the local ODE system will have the size $p$. 


\subsection{Application of Krylov IIF schemes}

Finally we have the ODE system resulting from the $P^{1}(24)$ or $P^{2}$ (26) DG spatial discretization:

$$
\vec{V}_{m}^{\prime}(t)=D \sum_{l=m, i, j, k} \widetilde{\mathbf{W}}_{l} \vec{V}_{l}(t)+\overrightarrow{\widetilde{F}}_{m}\left(\vec{V}_{m}\right), \quad m=1, \ldots, N,
$$

where $\widetilde{\mathbf{W}}_{l}=\mathbf{Q}_{m}^{-1} \mathbf{W}_{l}, \vec{F}_{m}=\mathbf{Q}_{m}^{-1} \vec{F}_{m} \cdot \mathbf{Q}_{m}^{-1}$ and $\widetilde{\mathbf{W}}_{l}$ are mesh-dependent data and they do not depend on the numerical solution $u$. So we pre-calculate and store these data before the time evolution. Note that, the nonlinear term $\vec{F}_{m}\left(\vec{V}_{m}\right)$ considered in this paper arises from the nonlinear reaction or source terms and no spatial derivative is involved in it, so the nonlinear operator $\widetilde{F}_{m}$ is local, namely it only depends on the unknowns $\vec{V}_{m}$ on the element $\Delta_{m}$. For more general and complex PDEs in which the nonlinear terms involve spatial derivatives, the nonlinear operator $\vec{F}_{m}$ is not local any more. Hence implicit treatment of the global nonlinear operator leads to coupled nonlinear system which is expensive to solve. For such case, semi-implicit approach needs to be applied to reduce the computational cost. Again, this will be one of our future works.

Assembling the local systems (27), we get the global ODE system arising from the implicit DG spatial discretization for a reaction-diffusion equation on a triangular mesh

$$
U^{\prime}(t)=\mathbf{A} U+\mathbf{F}(U)
$$

where $U=\left(V_{1}^{T}, V_{2}^{T}, \ldots, V_{N}^{T}\right)^{T}, \mathbf{A}$ is a $p \cdot N \times p \cdot N$ sparse matrix with block structures, where $p$ is the degrees of freedom on each triangular element. The nonzero elements in $\mathbf{A}$ are $p \times p$ sub-matrices $D \widetilde{\mathbf{W}}_{l}$ distributed on corresponding locations, and

$$
\mathbf{F}(U)=\left(\overrightarrow{\widetilde{F}}_{1}\left(\vec{V}_{1}\right)^{T}, \overrightarrow{\widetilde{F}}_{2}\left(\vec{V}_{2}\right)^{T}, \ldots, \overrightarrow{\widetilde{F}}_{N}\left(\vec{V}_{N}\right)^{T}\right)^{T} .
$$

Remark. The sparse matrix $\mathbf{A}$ is the $P^{1}$ or $P^{2}$ DG discretization (14) of the Laplacian operator $D \nabla^{2}$. For the sparse matrix $\mathbf{A}$ we only store the nonzero elements (those $D \widetilde{\mathbf{W}}_{l}$ blocks) and their locations in the matrix [53]. In our implementation we avoid the operations involving zero elements in the sparse matrix $\mathbf{A}$.

Now for the ODE system (28), we can directly apply the Krylov IIF methods (10) for the time evolution. In our numerical experiments, we apply the second order Krylov IIF method (11) for the $P^{1}$ DG spatial discretization, and the third order Krylov IIF method (12) for the $P^{2}$ DG spatial discretization to achieve consistent spatial-temporal accuracy orders. As the original IIF methods, the Krylov IIF methods will result in a local nonlinear algebraic system on every triangular element. The number of algebraic equations of the local system is the product of the degrees of freedom $p$ and the number of equations in the PDEs system. We use the Newton method to solve the small nonlinear algebraic system on every triangular element. In the Newton iterations to compute $U_{n+1}$, we use the numerical values $U_{n}$ at time step $t_{n}$ as the initial guess. And the threshold value for judging Newton iteration convergence is set to be $10^{-15}$. Newton iterations converge very fast for all numerical examples in this paper, since the nonlinear systems are local and have small sizes. While iteration numbers are larger for larger time step sizes (due to bigger distances between the initial guess $U_{n}$ and the solution $U_{n+1}$ ), the average iteration number is about 5 for nonlinear numerical examples in the next section (the iteration number is just 2 for linear problems).

\section{Numerical experiments}

In this section we present numerical examples to show the stability, accuracy and efficiency of the Krylov IIF methods in resolving the stiffness of the DG spatial operator for reaction-diffusion PDEs on 2D triangular meshes. The methods are firstly tested on a set of problems with exact solutions. Then application of the method to long-time simulation of a mathematical model which describes the dorsal-ventral pattern formation during the zebrafish embryo development will be shown. From numerical experiments we can observe that large time step sizes are achieved in numerical computations of these parabolic PDEs by the DG method.

In this paper, all of the time-dependent reaction-diffusion systems we considered are subject to no-flux boundary conditions. If the element edge $e_{l}$ of $\Delta_{m}$ is aligned with the domain boundary $\partial \Omega$, we take $\left.u^{i n}\right|_{e_{l}}=\left.u^{e x t}\right|_{e_{l}}$, and $\left.(\nabla u)^{i n}\right|_{e_{l}} \cdot \vec{n}_{e_{l}}=$ $\left.(\nabla u)^{\text {ext }}\right|_{e_{l}} \cdot \vec{n}_{e_{l}}=0$ in the numerical fluxes (15)-(17). Hence we have

$$
\left.\hat{u}\right|_{e_{l}}=\left.u^{i n}\right|_{e_{l}},\left.\quad \widetilde{\nabla u}\right|_{e_{l}} \cdot \vec{n}_{e_{l}}=0
$$

in scheme (14).

All computations in this paper are performed on a $2.39 \mathrm{GHz}, 8 \mathrm{~GB}$ RAM Linux computer.

\subsection{Numerical examples with exact solutions}

In this subsection, we perform convergence studies for the second and third order Krylov IIF methods which are applied to the $P^{1}$ and $P^{2}$ DG spatial discretizations respectively on triangular meshes, as shown in Fig. 1(a) which is a coarse one with 56 

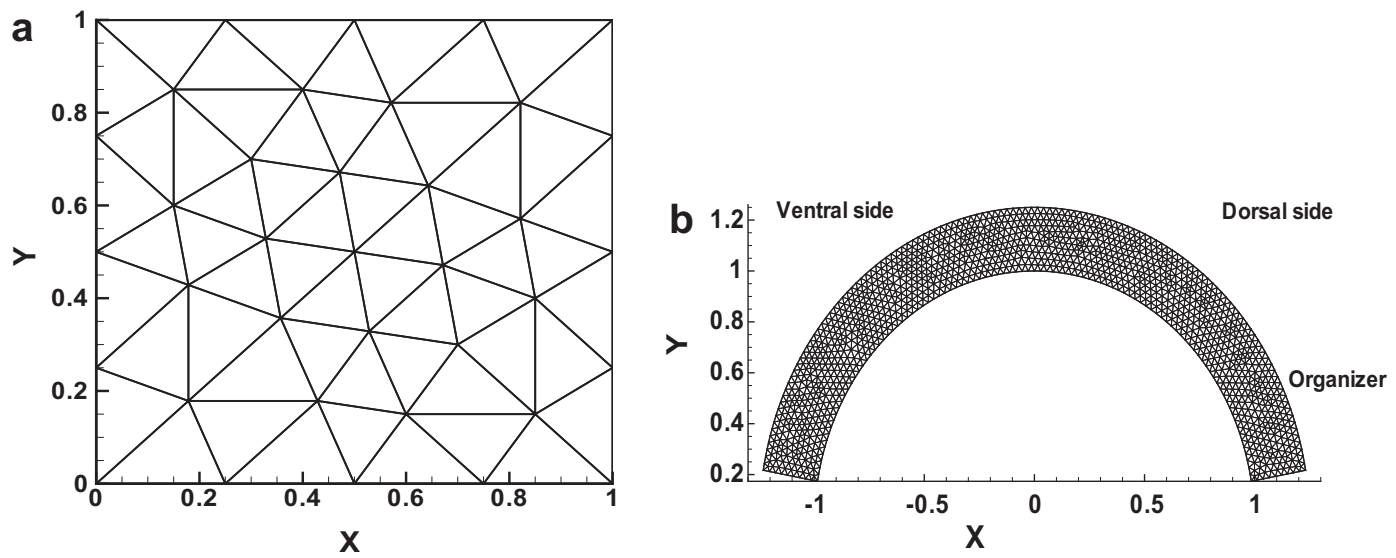

Fig. 1. (a) A coarse mesh with 56 triangles in the convergence studies in Section 4.1; (b) a coarse mesh with 2415 triangles in the numerical simulations for the zebrafish model in Section 4.2 .

elements. The refinement of the triangular meshes is done in a uniform way, namely by cutting each triangle into four smaller similar ones.

To show the stiffness of the DG spatial discretization matrix $\mathbf{A}$ for the diffusion operator $\nabla^{2}$, we study the eigenvalues and condition numbers related to $\mathbf{A}$. In Table 1, we list the largest negative eigenvalue $\lambda_{0}(\mathbf{A})$ of the $P^{1}$ or $P^{2}$ DG discretization matrices $\mathbf{A}$ for the diffusion operator $\nabla^{2}$ on the domain $[0,1]^{2}$ with no-flux boundary conditions, on successively refined meshes. The matrices $\mathbf{A}$ have a 0 eigenvalue due to the no-flux boundary condition. We can see that the matrices $\mathbf{A}$ have quite large magnitude eigenvalues for not so refined meshes. If an regular implicit method is used for the time discretization (e.g. the backward Euler method), a linear system with the coefficient matrix $I-\Delta t \mathbf{A}$ needs to be solved. In Table 1, we also list the condition numbers of the matrices $I-\Delta t \mathbf{A}$ for successively refined spatial-temporal meshes, with $\Delta t=h_{\min }$. These condition numbers are quite large for not so refined 2D meshes. Next we will show that the Krylov IIF methods developed in this paper work quite well for this DG spatial discretization.

Example 1. Consider the two-dimensional linear parabolic problem

$$
\left\{\begin{array}{l}
u_{t}=u_{x x}+u_{y y}+a\left(2 \pi^{2} e^{-t} \cos (\pi x) \cos (\pi y)-u\right), \quad(x, y) \in(0,1) \times(0,1) \\
u(x, y, 0)=\cos (\pi x) \cos (\pi y)
\end{array}\right.
$$

with no-flux boundary conditions. $a$ is a constant. We use this linear problem to test the linear stability of our methods. We consider the $a=0$ and $a=1$ cases. $a=0$ corresponds to a pure diffusion problem, i.e., $\mathbf{F}(U(t))=0$ in $(1)$. $a=1$ corresponds to a linear reaction-diffusion problem.

(Case 1, $a=0$.) This is a pure diffusion problem with the exact solution $u(x, y, t)=e^{-2 \pi^{2} t} \cos (\pi x) \cos (\pi y)$. The computation is carried up to $T=0.6$ with $M=25$ at which the $L^{1}, L^{2}$ and $L^{\infty}$ errors are measured. The time step size $\Delta t=h_{\min }$. CPU time, errors and order of accuracy for the second order Krylov IIF method with $P^{1}$ DG spatial discretization are reported in Table 2 , and for the third order Krylov IIF method with $P^{2}$ DG spatial discretization are reported in Table 3 . We can observe that we obtain desired accuracy orders for both cases. In the Tables, Nee is the number of triangular elements of the computational meshes, and $N=3 \cdot$ Nee $\left(P^{1}\right.$ case) or $N=6 \cdot$ Nee ( $P^{2}$ case) is the number of unknown degrees of freedom in the spatial direc-

Table 1

Eigenvalue and condition number study. $\lambda_{0}(\mathbf{A})$ is the largest negative eigenvalue of $P^{1}$ or $P^{2}$ DG discretization matrices $\mathbf{A}$ for the diffusion operator $\nabla^{2}$ on the domain $[0,1]^{2}$ with no-flux boundary conditions, on successively refined meshes. cond $(I-\Delta t \mathbf{A})$ are condition numbers for the linear operator $I-\Delta t \mathbf{A}$, which will arise if an implicit treatment of the diffusion term is used. Time step size $\Delta t=h_{\min }$ is used. Nee is the number of triangular elements of the computational meshes.

\begin{tabular}{|c|c|c|c|c|c|}
\hline \multirow[t]{2}{*}{$\mathrm{Nee}$} & \multirow[t]{2}{*}{$\Delta t$} & \multicolumn{2}{|l|}{$P^{1}$} & \multicolumn{2}{|l|}{$P^{2}$} \\
\hline & & $\operatorname{cond}(I-\Delta t \mathbf{A})$ & $\lambda_{0}(\mathbf{A})$ & $\operatorname{cond}(I-\Delta t \mathbf{A})$ & $\lambda_{0}(\mathbf{A})$ \\
\hline 14 & 0.2079 & $2.7549 \mathrm{E}+03$ & $-3.1490 \mathrm{E}+03$ & $2.2734 \mathrm{E}+04$ & $-4.2638 \mathrm{E}+03$ \\
\hline 56 & 0.1039 & $4.8715 \mathrm{E}+03$ & $-1.3522 \mathrm{E}+04$ & $3.6438 \mathrm{E}+04$ & $-1.7467 \mathrm{E}+04$ \\
\hline 224 & 0.0520 & $9.0636 \mathrm{E}+03$ & $-5.5484 \mathrm{E}+04$ & $6.3633 \mathrm{E}+04$ & $-7.0285 \mathrm{E}+04$ \\
\hline 896 & 0.0260 & $1.9658 \mathrm{E}+04$ & $-2.2407 \mathrm{E}+05$ & $1.3349 \mathrm{E}+05$ & $-2.8164 \mathrm{E}+05$ \\
\hline 3584 & 0.0130 & $3.6801 \mathrm{E}+04$ & $-8.9920 \mathrm{E}+05$ & $2.4045 \mathrm{E}+05$ & $-1.1277 \mathrm{E}+06$ \\
\hline
\end{tabular}


Table 2

Example 1, case 1 . CPU time, error, and order of accuracy of the second order Krylov IIF method with $P^{1}$ DG spatial discretization. Final time $T=0.6$.

\begin{tabular}{|c|c|c|c|c|c|c|c|c|}
\hline Nee & $N$ & $\mathrm{CPU}(\mathrm{s})$ & $L_{1}$ error & Order & $L_{2}$ error & Order & $L_{\infty}$ error & Order \\
\hline 14 & 42 & 0.00 & $2.60 \mathrm{E}-06$ & - & $3.17 \mathrm{E}-06$ & - & $6.47 \mathrm{E}-06$ & - \\
\hline 56 & 168 & 0.02 & $1.20 \mathrm{E}-06$ & 1.12 & $1.48 \mathrm{E}-06$ & 1.10 & $2.70 \mathrm{E}-06$ & 1.26 \\
\hline 224 & 672 & 0.12 & $3.70 \mathrm{E}-07$ & 1.70 & $4.54 \mathrm{E}-07$ & 1.70 & $9.00 \mathrm{E}-07$ & 1.59 \\
\hline 896 & 2688 & 0.69 & $9.86 \mathrm{E}-08$ & 1.91 & $1.21 \mathrm{E}-07$ & 1.91 & $2.41 \mathrm{E}-07$ & 1.90 \\
\hline 3584 & 10752 & 5.24 & $2.49 \mathrm{E}-08$ & 1.98 & $3.06 \mathrm{E}-08$ & 1.98 & $6.09 \mathrm{E}-08$ & 1.98 \\
\hline 14336 & 43008 & 40.23 & $6.24 \mathrm{E}-09$ & 2.00 & $7.67 \mathrm{E}-09$ & 2.00 & $1.52 \mathrm{E}-08$ & 2.00 \\
\hline 57344 & 172032 & 368.65 & $1.56 \mathrm{E}-09$ & 2.00 & $1.92 \mathrm{E}-09$ & 2.00 & $3.81 \mathrm{E}-09$ & 2.00 \\
\hline
\end{tabular}

Table 3

Example 1, case 1 . CPU time, error, and order of accuracy of the third order Krylov IIF method with $P^{2}$ DG spatial discretization. Final time $T=0.6$.

\begin{tabular}{|c|c|c|c|c|c|c|c|c|}
\hline Nee & $N$ & $\mathrm{CPU}(\mathrm{s})$ & $L_{1}$ error & Order & $L_{2}$ error & Order & $L_{\infty}$ error & Order \\
\hline 14 & 84 & 0.01 & $3.46 \mathrm{E}-07$ & - & $4.02 \mathrm{E}-07$ & - & $6.02 \mathrm{E}-07$ & - \\
\hline 56 & 336 & 0.05 & $1.12 \mathrm{E}-08$ & 4.95 & $1.47 \mathrm{E}-08$ & 4.78 & $6.34 \mathrm{E}-08$ & 3.25 \\
\hline 224 & 1344 & 0.26 & $8.19 \mathrm{E}-10$ & 3.78 & $1.06 \mathrm{E}-09$ & 3.79 & $8.25 \mathrm{E}-09$ & 2.94 \\
\hline 896 & 5376 & 1.99 & $7.24 \mathrm{E}-11$ & 3.50 & $9.38 \mathrm{E}-11$ & 3.50 & $1.04 \mathrm{E}-09$ & 2.99 \\
\hline 3584 & 21504 & 16.05 & $8.11 \mathrm{E}-12$ & 3.16 & $1.06 \mathrm{E}-11$ & 3.14 & $1.30 \mathrm{E}-10$ & 3.00 \\
\hline 14336 & 86016 & 134.62 & $9.83 E-13$ & 3.04 & $1.30 \mathrm{E}-12$ & 3.03 & $1.63 \mathrm{E}-11$ & 3.00 \\
\hline 57344 & 344064 & 994.91 & $1.25 \mathrm{E}-13$ & 2.98 & $1.65 \mathrm{E}-13$ & 2.98 & $2.06 \mathrm{E}-12$ & 2.98 \\
\hline
\end{tabular}

tion. If the original IIF schemes are used, it is computationally prohibitive to compute the matrix exponential for such huge $N \times N$ matrices, especially when the spatial mesh is refined. For the Krylov IIF schemes in this paper, we only need to compute the matrix exponentials of $25 \times 25$ matrices, which are independent of the degrees of freedom $N$. As shown in the CPU time of the numerical tables, we can see that the computations are very efficient. When the spatial mesh is refined, the number of degrees of freedom $N$ increase 4 times and the total time steps double. From Tables 2 and 3, we see that the CPU time approximately increases 8 times when the mesh is refined once. So the CPU time approximately linearly depends on the number of degrees of freedom. Also, we obtain a clean second and third order accuracy in the computations for a fixed Krylov space dimension $M=25$ for all meshes, and this indicates that the errors generated by the Krylov subspace approximations are much smaller than the truncation errors of the numerical schemes. This is also confirmed by the numerical results in Table 4, in which we show numerical errors if different dimensions $M$ of the Krylov subspace are used in the second order Krylov IIF method with $P^{1}$ DG spatial discretization, for a fixed spatial mesh. We can see that the numerical errors are comparable for smaller values of $M$ and larger ones. So the numerical errors shown in Table 4 are mainly due to the truncation errors of the DG spatial discretizations (14)(the temporal truncation errors from the IIF part (5) are 0 since this problem only has the linear diffusion part), and the numerical errors by the Krylov subspace approximations are negligible in this example. We will further perform theoretical error analysis on this in our future work. The numerical results also show that even for this parabolic PDE, we can use a large time step size proportional to the spatial grid size for a stable and accurate computation. Actually for this problem which only has linear diffusion terms, in the time direction the Krylov IIF method can evolve the linear DG spatial discretization operator almost "exactly" up to the numerical errors of the Krylov subspace approximations. This is shown in both Tables 4 and 5. From these two tables, we can see that even if we use a very large time step size $\Delta t=0.6$, namely we just use one time-step to reach the final time $T$, we can still obtain comparable numerical errors as those in the computations by using $\Delta t=h_{\text {min }}$ and a clear second order accuracy as shown in Table 5.

(Case $2, a=1$.) This is a linear reaction-diffusion equation with the exact solution $u(x, y, t)=e^{-t} \cos (\pi x) \cos (\pi y)$. Unlike the case 1 which is a pure diffusion problem, the numerical errors from temporal IIF discretizations of the reaction term will play a role since $\mathbf{F}(U(t))$ in (1) is not zero any more. The computation is carried up to $T=2.0$ with $M=25$ at which the $L^{1}, L^{2}$ and $L^{\infty}$ errors are measured. We test the linear stability of our methods by increasing the time step size successively as $\Delta t=h_{\text {min }}$, $\Delta t=2 h_{\text {min }}$, and $\Delta t=4 h_{\text {min }}$. CPU time, errors and order of accuracy for the second order Krylov IIF method with $P^{1}$ DG spatial discretization are reported in Table 6, and for the third order Krylov IIF method with $P^{2}$ DG spatial discretization are reported in Table 7. We can observe that we obtained desired accuracy orders for all cases. In the tables, Nee is the number of

Table 4

Example 1, case 1 . Numerical errors if different dimensions $M$ of the Krylov subspace are used in the second order Krylov IIF method with $P^{1}$ DG spatial discretization. Final time $T=0.6$. Two different time step sizes $\Delta t=0.6$ and $\Delta t=h_{\min }$ are used. The spatial mesh is the one which has Nee $=3584$ triangles.

\begin{tabular}{|c|c|c|c|c|c|c|}
\hline \multirow[t]{2}{*}{$M$} & \multicolumn{3}{|l|}{$\Delta t=0.6$} & \multicolumn{3}{|l|}{$\Delta t=h_{\min }$} \\
\hline & $L_{1}$ error & $L_{2}$ error & $L_{\infty}$ error & $L_{1}$ error & $L_{2}$ error & $L_{\infty}$ error \\
\hline 10 & $3.06 \mathrm{E}-08$ & $3.77 \mathrm{E}-08$ & $7.74 \mathrm{E}-08$ & $2.45 \mathrm{E}-08$ & $3.02 \mathrm{E}-08$ & $5.98 \mathrm{E}-08$ \\
\hline 25 & $2.69 \mathrm{E}-08$ & $3.32 \mathrm{E}-08$ & $6.78 \mathrm{E}-08$ & $2.49 \mathrm{E}-08$ & $3.06 \mathrm{E}-08$ & $6.09 \mathrm{E}-08$ \\
\hline 100 & $2.44 \mathrm{E}-08$ & $3.01 \mathrm{E}-08$ & $5.99 \mathrm{E}-08$ & $2.49 \mathrm{E}-08$ & $3.06 \mathrm{E}-08$ & $6.09 \mathrm{E}-08$ \\
\hline 250 & $2.48 \mathrm{E}-08$ & $3.05 \mathrm{E}-08$ & $6.03 \mathrm{E}-08$ & $2.49 \mathrm{E}-08$ & $3.06 \mathrm{E}-08$ & $6.09 \mathrm{E}-08$ \\
\hline
\end{tabular}


Table 5

Example 1, case 1 . Error, and order of accuracy of the second order Krylov IIF method with $P^{1}$ DG spatial discretization. Final time $T=0.6$. A large time step $\Delta t=0.6$ is used, namely, only one step to the final time for this diffusion problem.

\begin{tabular}{|c|c|c|c|c|c|c|c|}
\hline Nee & $N$ & $L_{1}$ error & Order & $L_{2}$ error & Order & $L_{\infty}$ error & Order \\
\hline 14 & 42 & $2.60 \mathrm{E}-06$ & - & $3.18 \mathrm{E}-06$ & - & $6.50 \mathrm{E}-06$ & - \\
\hline 56 & 168 & $1.20 \mathrm{E}-06$ & 1.12 & $1.48 \mathrm{E}-06$ & 1.11 & $2.71 \mathrm{E}-06$ & 1.26 \\
\hline 224 & 672 & $3.69 \mathrm{E}-07$ & 1.70 & $4.54 \mathrm{E}-07$ & 1.70 & $8.99 \mathrm{E}-07$ & 1.59 \\
\hline 896 & 2688 & $1.11 \mathrm{E}-07$ & 1.73 & $1.37 \mathrm{E}-07$ & 1.73 & $2.74 \mathrm{E}-07$ & 1.72 \\
\hline 3584 & 10752 & $2.69 \mathrm{E}-08$ & 2.04 & $3.32 \mathrm{E}-08$ & 2.04 & $6.78 \mathrm{E}-08$ & 2.01 \\
\hline 14336 & 43008 & $6.64 \mathrm{E}-09$ & 2.02 & $8.19 \mathrm{E}-09$ & 2.02 & $1.69 \mathrm{E}-08$ & 2.00 \\
\hline 57344 & 172032 & $1.70 \mathrm{E}-09$ & 1.96 & $2.10 \mathrm{E}-09$ & 1.96 & $4.35 \mathrm{E}-09$ & 1.96 \\
\hline
\end{tabular}

Table 6

Example 1, case 2. CPU time, error, and order of accuracy of the second order Krylov IIF method with $P^{1}$ DG spatial discretization. Final time $T=2$.

\begin{tabular}{|c|c|c|c|c|c|c|c|c|}
\hline $\mathrm{Nee}$ & $N$ & $\mathrm{CPU}(\mathrm{s})$ & $L_{1}$ error & Order & $L_{2}$ error & Order & $L_{\infty}$ error & Order \\
\hline \multicolumn{9}{|c|}{$\Delta t=h_{\min }$} \\
\hline 14 & 42 & 0.01 & $2.10 \mathrm{E}-02$ & - & $2.62 \mathrm{E}-02$ & - & $9.67 \mathrm{E}-02$ & - \\
\hline 56 & 168 & 0.10 & $7.60 \mathrm{E}-03$ & 1.47 & $9.56 \mathrm{E}-03$ & 1.46 & $3.33 \mathrm{E}-02$ & 1.54 \\
\hline 224 & 672 & 0.46 & $2.24 \mathrm{E}-03$ & 1.76 & $2.79 \mathrm{E}-03$ & 1.78 & $9.35 \mathrm{E}-03$ & 1.83 \\
\hline 896 & 2688 & 2.82 & $8.25 \mathrm{E}-04$ & 1.44 & $1.02 \mathrm{E}-03$ & 1.46 & $3.04 \mathrm{E}-03$ & 1.62 \\
\hline 3584 & 10752 & 21.95 & $2.07 \mathrm{E}-04$ & 1.99 & $2.56 \mathrm{E}-04$ & 1.99 & $7.80 \mathrm{E}-04$ & 1.96 \\
\hline 14336 & 43008 & 171.41 & $5.23 \mathrm{E}-05$ & 1.99 & $6.45 \mathrm{E}-05$ & 1.99 & $1.99 \mathrm{E}-04$ & 1.97 \\
\hline 57344 & 172032 & 1662.97 & $1.33 \mathrm{E}-05$ & 1.98 & $1.64 \mathrm{E}-05$ & 1.98 & $5.12 \mathrm{E}-05$ & 1.96 \\
\hline \multicolumn{9}{|c|}{$\Delta t=2 h_{\min }$} \\
\hline 14 & 42 & 0.00 & $1.00 \mathrm{E}-01$ & - & $1.30 \mathrm{E}-01$ & - & $3.40 \mathrm{E}-01$ & - \\
\hline 56 & 168 & 0.05 & $2.33 \mathrm{E}-02$ & 2.14 & $2.87 \mathrm{E}-02$ & 2.15 & $7.57 \mathrm{E}-02$ & 2.18 \\
\hline 224 & 672 & 0.27 & $9.22 \mathrm{E}-03$ & 1.34 & $1.14 \mathrm{E}-02$ & 1.34 & $2.70 \mathrm{E}-02$ & 1.49 \\
\hline 896 & 2688 & 1.58 & $2.68 \mathrm{E}-03$ & 1.78 & $3.30 \mathrm{E}-03$ & 1.78 & $7.63 \mathrm{E}-03$ & 1.82 \\
\hline 3584 & 10752 & 11.89 & $9.44 \mathrm{E}-04$ & 1.50 & $1.17 \mathrm{E}-03$ & 1.50 & $2.59 \mathrm{E}-03$ & 1.56 \\
\hline 14336 & 43008 & 89.35 & $2.37 \mathrm{E}-04$ & 2.00 & $2.92 \mathrm{E}-04$ & 2.00 & $6.52 \mathrm{E}-04$ & 1.99 \\
\hline 57344 & 172032 & 741.47 & $5.95 \mathrm{E}-05$ & 1.99 & $7.34 \mathrm{E}-05$ & 1.99 & $1.65 \mathrm{E}-04$ & 1.98 \\
\hline \multicolumn{9}{|c|}{$\Delta t=4 h_{\min }$} \\
\hline 56 & 168 & 0.03 & $1.00 \mathrm{E}-01$ & - & $1.30 \mathrm{E}-01$ & - & $2.90 \mathrm{E}-01$ & - \\
\hline 224 & 672 & 0.16 & $2.44 \mathrm{E}-02$ & 2.06 & $3.01 \mathrm{E}-02$ & 2.06 & $6.53 \mathrm{E}-02$ & 2.14 \\
\hline 896 & 2688 & 0.95 & $9.61 \mathrm{E}-03$ & 1.34 & $1.19 \mathrm{E}-02$ & 1.34 & $2.48 \mathrm{E}-02$ & 1.40 \\
\hline 3584 & 10752 & 6.63 & $2.80 \mathrm{E}-03$ & 1.78 & $3.45 \mathrm{E}-03$ & 1.78 & $7.17 \mathrm{E}-03$ & 1.79 \\
\hline 14336 & 43008 & 48.03 & $9.74 \mathrm{E}-04$ & 1.52 & $1.20 \mathrm{E}-03$ & 1.52 & $2.47 \mathrm{E}-03$ & 1.54 \\
\hline 57344 & 172032 & 409.27 & $2.44 \mathrm{E}-04$ & 2.00 & $3.01 \mathrm{E}-04$ & 2.00 & $6.19 \mathrm{E}-04$ & 2.00 \\
\hline
\end{tabular}

triangular elements of the computational meshes, and $N=3 \cdot$ Nee $\left(P^{1}\right.$ case $)$ or $N=6 \cdot$ Nee $\left(P^{2}\right.$ case $)$ is the number of unknown degrees of freedom in the spatial direction. As in the case 1, the Krylov IIF methods demonstrate excellent efficiency, accuracy and stability properties in this case. The stable and accurate computation results by using large time step sizes show that the methods have quite large linear stability region.

Example 2. Consider a two-dimensional nonlinear reaction-diffusion problem

$$
\left\{\begin{array}{l}
u_{t}=u_{x x}+u_{y y}-u^{2}+e^{-2 t} \cos ^{2}(\pi x) \cos ^{2}(\pi y)+\left(2 \pi^{2}-1\right) e^{-t} \cos (\pi x) \cos (\pi y), \quad(x, y) \in(0,1) \times(0,1) \\
u(x, y, 0)=\cos (\pi x) \cos (\pi y),
\end{array}\right.
$$

with no-flux boundary conditions. The exact solution is $u(x, y, t)=e^{-t} \cos (\pi x) \cos (\pi y)$. The computation is carried up to $T=2.0$ with $M=25$ at which the $L^{1}, L^{2}$ and $L^{\infty}$ errors are measured. The time step size $\Delta t=h_{\text {min. }}$ CPU time, errors and order of accuracy for the second order Krylov IIF method with $P^{1}$ DG spatial discretization are reported in Table 8 , and for the third order Krylov IIF method with $P^{2}$ DG spatial discretization are reported in Table 9. Again we obtained desired accuracy orders for both cases. In the tables, Nee is the number of triangular elements of the computational meshes, and $N=3 \cdot N e e\left(P^{1}\right.$ case $)$ or $N=6 \cdot$ Nee ( $P^{2}$ case) is the number of unknown degrees of freedom in the spatial direction. For this example with a nonlinear reaction term, we still draw the same conclusion as in the last example, from the numerical convergence study about the efficiency, accuracy and stability properties of the Krylov IIF methods.

\subsection{Application to a morphogenesis system}

Many mathematical models in developmental biology take the form of reaction-diffusion Eq. (13). In such systems, both diffusion and reaction terms are often very stiff. And long time numerical simulations are often needed for these systems. 
Table 7

Example 1, case 2. CPU time, error, and order of accuracy of the third order Krylov IIF method with $P^{2}$ DG spatial discretization. Final time $T=2$.

\begin{tabular}{|c|c|c|c|c|c|c|c|c|}
\hline Nee & $N$ & $\mathrm{CPU}(\mathrm{s})$ & $L_{1}$ error & Order & $L_{2}$ error & Order & $L_{\infty}$ error & Order \\
\hline \multicolumn{9}{|c|}{$\Delta t=h_{\min }$} \\
\hline 14 & 84 & 0.07 & $1.49 \mathrm{E}-02$ & - & $1.84 \mathrm{E}-02$ & - & $4.54 \mathrm{E}-02$ & - \\
\hline 56 & 336 & 0.31 & $5.11 \mathrm{E}-03$ & 1.55 & $6.29 \mathrm{E}-03$ & 1.54 & $1.31 \mathrm{E}-02$ & 1.80 \\
\hline 224 & 1344 & 1.81 & $9.44 \mathrm{E}-04$ & 2.44 & $1.16 \mathrm{E}-03$ & 2.43 & $2.36 \mathrm{E}-03$ & 2.47 \\
\hline 896 & 5376 & 14.21 & $1.99 \mathrm{E}-04$ & 2.25 & $2.45 \mathrm{E}-04$ & 2.25 & $4.92 \mathrm{E}-04$ & 2.26 \\
\hline 3584 & 21504 & 115.10 & $2.71 \mathrm{E}-05$ & 2.88 & $3.34 \mathrm{E}-05$ & 2.88 & $6.70 \mathrm{E}-05$ & 2.88 \\
\hline 14336 & 86016 & 940.71 & $3.59 \mathrm{E}-06$ & 2.91 & $4.43 \mathrm{E}-06$ & 2.91 & $8.88 \mathrm{E}-06$ & 2.91 \\
\hline 57344 & 344064 & 7898.45 & $4.76 \mathrm{E}-07$ & 2.92 & $5.87 \mathrm{E}-07$ & 2.92 & $1.18 \mathrm{E}-06$ & 2.92 \\
\hline \multicolumn{9}{|c|}{$\Delta t=2 h_{\min }$} \\
\hline 14 & 84 & 0.03 & $7.87 \mathrm{E}-02$ & - & $9.73 E-02$ & - & 0.21 & - \\
\hline 56 & 336 & 0.16 & $1.51 \mathrm{E}-02$ & 2.38 & $1.86 \mathrm{E}-02$ & 2.39 & $3.78 \mathrm{E}-02$ & 2.48 \\
\hline 224 & 1344 & 0.97 & $5.11 \mathrm{E}-03$ & 1.56 & $6.31 \mathrm{E}-03$ & 1.56 & $1.27 \mathrm{E}-02$ & 1.58 \\
\hline 896 & 5376 & 7.55 & $9.44 \mathrm{E}-04$ & 2.44 & $1.16 \mathrm{E}-03$ & 2.44 & $2.33 \mathrm{E}-03$ & 2.44 \\
\hline 3584 & 21504 & 58.61 & $1.99 \mathrm{E}-04$ & 2.25 & $2.45 \mathrm{E}-04$ & 2.25 & $4.91 \mathrm{E}-04$ & 2.25 \\
\hline 14336 & 86016 & 500.34 & $2.71 \mathrm{E}-05$ & 2.88 & $3.34 \mathrm{E}-05$ & 2.88 & $6.69 \mathrm{E}-05$ & 2.88 \\
\hline 57344 & 344064 & 4288.04 & $3.59 \mathrm{E}-06$ & 2.91 & $4.43 \mathrm{E}-06$ & 2.91 & $8.86 \mathrm{E}-06$ & 2.92 \\
\hline \multicolumn{9}{|c|}{$\Delta t=4 h_{\min }$} \\
\hline 56 & 336 & 0.09 & $7.89 \mathrm{E}-02$ & - & $9.73 \mathrm{E}-02$ & - & $2.00 \mathrm{E}-01$ & - \\
\hline 224 & 1344 & 0.54 & $1.51 \mathrm{E}-02$ & 2.38 & $1.86 \mathrm{E}-02$ & 2.38 & $3.73 \mathrm{E}-02$ & 2.39 \\
\hline 896 & 5376 & 4.03 & $5.12 \mathrm{E}-03$ & 1.56 & $6.31 \mathrm{E}-03$ & 1.56 & $1.26 \mathrm{E}-02$ & 1.56 \\
\hline 3584 & 21504 & 30.85 & $9.44 \mathrm{E}-04$ & 2.44 & $1.16 \mathrm{E}-03$ & 2.44 & $2.33 \mathrm{E}-03$ & 2.44 \\
\hline 14336 & 86016 & 249.01 & $1.99 \mathrm{E}-04$ & 2.25 & $2.45 \mathrm{E}-04$ & 2.25 & $4.91 \mathrm{E}-04$ & 2.25 \\
\hline 57344 & 344064 & 1971.68 & $2.71 \mathrm{E}-05$ & 2.88 & $3.34 \mathrm{E}-05$ & 2.88 & $6.69 \mathrm{E}-05$ & 2.88 \\
\hline
\end{tabular}

Table 8

Example 2. CPU time, error, and order of accuracy of the second order Krylov IIF method with $P^{1}$ DG spatial discretization. Final time $T=2$.

\begin{tabular}{|c|c|c|c|c|c|c|c|c|}
\hline Nee & $N$ & $\mathrm{CPU}(\mathrm{s})$ & $L_{1}$ error & Order & $L_{2}$ error & Order & $L_{\infty}$ error & Order \\
\hline 14 & 42 & 0.06 & 0.59 & - & 0.59 & - & 0.72 & - \\
\hline 56 & 168 & 0.11 & $1.45 \mathrm{E}-02$ & 5.34 & $1.70 \mathrm{E}-02$ & 5.12 & $4.87 \mathrm{E}-02$ & 3.88 \\
\hline 224 & 672 & 0.52 & $2.44 \mathrm{E}-03$ & 2.58 & $3.02 \mathrm{E}-03$ & 2.50 & $9.93 \mathrm{E}-03$ & 2.29 \\
\hline 896 & 2688 & 3.19 & $8.72 \mathrm{E}-04$ & 1.48 & $1.08 \mathrm{E}-03$ & 1.49 & $3.19 E-03$ & 1.64 \\
\hline 3584 & 10752 & 24.60 & $2.19 \mathrm{E}-04$ & 1.99 & $2.70 \mathrm{E}-04$ & 1.99 & $8.18 \mathrm{E}-04$ & 1.96 \\
\hline 14336 & 43008 & 197.79 & $5.52 \mathrm{E}-05$ & 1.99 & $6.81 \mathrm{E}-05$ & 1.99 & $2.09 \mathrm{E}-04$ & 1.97 \\
\hline 57344 & 172032 & 1670.53 & $1.40 \mathrm{E}-05$ & 1.98 & $1.72 \mathrm{E}-05$ & 1.98 & $5.36 \mathrm{E}-05$ & 1.96 \\
\hline
\end{tabular}

Hence robust and accurate methods which permit large time step sizes are desired. As demonstrated in one-dimensional systems [48], the IIF methods are quite efficient for such applications. In this section, we apply the second order Krylov IIF method with $P^{1}$ DG spatial discretization on a triangular mesh to a model for the study of embryonic patterning. The model is re-defined on a two dimensional domain which has a realistic shape as part of the embryo during its development.

One of the central problems in developmental biology is how uniform fields of cells are transformed into tissues with highly specialized cell types at distinct anatomical positions. In this process, diffusible morphogens produced by certain cells pattern the surrounding tissue through interactions with certain proteins on the cell membrane. Morphogens are signaling molecules that, when bound to cell receptors, assign different cell fates at different concentrations. This role of morphogens has been the prevailing thought in tissue patterning for over half a century; but only recently have there been sufficient experimental data for us to begin to understand how various morphogens interact and patterns emerge. Now, mathematical modelling, analysis and computations have been very helpful to understand and identify underline biological mechanisms in morphogenesis systems [39,40].

The example we are considering is a system of reaction-diffusion equations arising from mathematical modeling of a morphogenesis problem during the dorsal-ventral patterning of zebrafish embryo development [67]. The model predicts that the dorsal organizer of the zebrafish embryo plays a key role in forming a stable non-homogeneous morphogen gradient, and the prediction agrees well with the existing biological experiments. First we briefly describe the reaction-diffusion model in the following.

Consider the zebrafish embryo from the end of blastula period to the middle of gastrula period (approximately 4.5-7.5 $\mathrm{h}$ after fertilization). We are interested in the patterning of the dorsal-ventral tissues. On a two dimensional approximation, the dorsal-ventral region has a shape as shown in Fig. 1(b), with the dorsal organizer being located at the corner of the dorsal region. The whole domain $\Omega$ can be represented by

$$
\Omega=\left\{(x, y) \mid r_{\min }^{2} \leqslant x^{2}+y^{2} \leqslant r_{\max }^{2}, \cos 170^{\circ} \leqslant \frac{x}{\sqrt{x^{2}+y^{2}}} \leqslant \cos 10^{\circ}\right\}
$$


Table 9

Example 2. CPU time, error, and order of accuracy of the third order Krylov IIF method with $P^{2}$ DG spatial discretization. Final time $T=2$.

\begin{tabular}{|c|c|c|c|c|c|c|c|c|}
\hline $\mathrm{Nee}$ & $N$ & $\mathrm{CPU}(\mathrm{s})$ & $L_{1}$ error & order & $L_{2}$ error & order & $L_{\infty}$ error & order \\
\hline 14 & 84 & 0.08 & 0.15 & - & 0.15 & - & 0.20 & - \\
\hline 56 & 336 & 0.35 & $6.57 \mathrm{E}-03$ & 4.49 & $7.98 \mathrm{E}-03$ & 4.23 & $1.83 \mathrm{E}-02$ & 3.46 \\
\hline 224 & 1344 & 2.08 & $1.01 \mathrm{E}-03$ & 2.69 & $1.25 \mathrm{E}-03$ & 2.67 & $2.58 \mathrm{E}-03$ & 2.83 \\
\hline 896 & 5376 & 16.18 & $2.10 \mathrm{E}-04$ & 2.27 & $2.59 \mathrm{E}-04$ & 2.27 & $5.28 \mathrm{E}-04$ & 2.29 \\
\hline 3584 & 21504 & 127.09 & $2.86 \mathrm{E}-05$ & 2.88 & $3.53 \mathrm{E}-05$ & 2.88 & $7.17 \mathrm{E}-05$ & 2.88 \\
\hline 14336 & 86016 & 1139.88 & $3.77 \mathrm{E}-06$ & 2.92 & $4.65 \mathrm{E}-06$ & 2.92 & $9.44 \mathrm{E}-06$ & 2.93 \\
\hline 57344 & 344064 & 8864.08 & $4.91 \mathrm{E}-07$ & 2.94 & $6.05 \mathrm{E}-07$ & 2.94 & $1.22 \mathrm{E}-06$ & 2.95 \\
\hline
\end{tabular}

and the dorsal organizer region $\Omega_{O}$ is

$$
\Omega_{0}=\left\{(x, y) \mid r_{\min }^{2} \leqslant x^{2}+y^{2} \leqslant r_{\text {max }}^{2}, \cos 30^{\circ} \leqslant \frac{x}{\sqrt{x^{2}+y^{2}}} \leqslant \cos 10^{\circ}\right\},
$$

where $r_{\min }$ and $r_{\max }$ are radii of the inner and outer boundaries.

Let $[L]$ and $[L R]$ denote the concentration of the morphogen BMP and the concentration of BMP-receptor complexes (to which BMP signaling is assumed to be proportional), respectively. The concentration of the free molecule Chordin (an inhibitor of BMP) and the concentration of BMP-Chordin complex are denoted by $[C]$ and $[L C]$, respectively. Let $R_{0}$ denote the total receptor concentration and let $D_{L}, D_{C}$, and $D_{L C}$ represent the three diffusion coefficients for BMP, Chordin, BMP-Chordin complexes, respectively. The values $k_{o n}, k_{\text {off }}, k_{d e g}, j_{\text {on }}, j_{\text {off }}$, and $\tau$ are the binding and degradation rates for BMP, Chordin, and their complexes. With this notation, the reaction-diffusion model that is formulated in [67] is as follows:

$$
\left\{\begin{array}{l}
\frac{\partial[L]}{\partial t}=D_{L} \nabla^{2}[L]-k_{o n}[L]\left(R_{0}-[L R]\right)+k_{o f f}[L R]-j_{o n}[L][C]+\left(j_{o f f}+\tau\right)[L C]+V_{L} \\
\frac{\partial[L R]}{\partial t}=k_{o n}[L]\left(R_{0}-[L R]\right)-\left(k_{o f f}+k_{\text {deg }}\right)[L R] \\
\frac{\partial[L C]}{\partial t}=D_{L C} \nabla^{2}[L C]+j_{o n}[L][C]-\left(j_{o f f}+\tau\right)[L C] \\
\frac{\partial[C]}{\partial t}=D_{C} \nabla^{2}[C]-j_{o n}[L][C]+j_{o f f}[L C]+V_{C}
\end{array}\right.
$$

where $V_{C}$ and $V_{L}$ are the production rates of molecules Chordin and BMP, respectively, defined by

$$
\begin{aligned}
& V_{C}=V_{C \min }+\frac{V_{C \max }-V_{C \min }}{1+\gamma_{C}[L R]}+ \begin{cases}V_{C o r g} e^{-a t}, & \text { if }(x, y) \in \Omega_{0} ; \\
0, & \text { otherwise. }\end{cases} \\
& V_{L}=V_{L \min }+\frac{V_{L \max }-V_{L \min }}{1+\gamma_{L}[L R]^{-1}}+V_{L m a t} e^{-b t} .
\end{aligned}
$$

The terms $V_{\text {Corg }} e^{-a t}$ and $V_{L m a t} e^{-b t}$ represent the maternal production rates of Chordin and BMP [67].

The system (31) is subjected to the no-flux boundary conditions

$$
\frac{\partial[L]}{\partial n}=\frac{\partial[L C]}{\partial n}=\frac{\partial[C]}{\partial n}=0 \text { for }(x, y) \in \partial \Omega .
$$

Parameters in the model are the following biological reasonable parameters [67]

$$
\begin{aligned}
& r_{\min }=0.028 \mathrm{~cm}, \quad r_{\max }=0.088 \mathrm{~cm}, \\
& D_{L}=D_{L C}=D_{C}=8.5 \times 10^{-7} \mathrm{~cm}^{2} \mathrm{~s}^{-1}, \quad k_{\text {on }}=0.4 \mu \mathrm{M}^{-1} \mathrm{~s}^{-1}, \\
& R_{0}=3.0 \mu \mathrm{M}, \quad k_{\text {off }}=4 \times 10^{-6} \mathrm{~s}^{-1}, \\
& j_{\text {on }}=10 \mu \mathrm{M}^{-1} \mathrm{~s}^{-1}, \quad j_{\text {off }}=1.0 \times 10^{-5} \mathrm{~s}^{-1}, \\
& \tau=0.01 \mathrm{~s}^{-1}, \quad k_{\text {deg }}=5.0 \times 10^{-4} \mathrm{~s}^{-1}, \\
& V_{C \min }=8.0 \times 10^{-4} \mu \mathrm{M} \mathrm{s}^{-1}, \quad V_{C \max }=8.0 \times 10^{-2} \mu \mathrm{M} \mathrm{s}^{-1}, \\
& V_{\text {Corg }}=6.68 \times 10^{-1} \mu \mathrm{M} \mathrm{s}^{-1}, \quad \gamma_{C}=10 \mu \mathrm{M}^{-1}, \\
& a=0.0167 \mathrm{~s}^{-1}, \quad V_{L \mathrm{~min}}=1.0 \times 10^{-5} \mu \mathrm{M} \mathrm{s}^{-1}, \\
& V_{L \max }=6.0 \times 10^{-3} \mu \mathrm{M} \mathrm{s}^{-1}, \quad \gamma_{L}=10 \mu \mathrm{M}, \\
& V_{L \text { mat }}=5.01 \times 10^{-2} \mu \mathrm{M} \mathrm{s}^{-1}, \quad b=0.0167 \mathrm{~s}^{-1},
\end{aligned}
$$


The system (31) can be non-dimensionalized with the following normalized quantities

$$
\begin{aligned}
& T=\frac{D}{r_{\min }^{2}} t, \quad X=\frac{x}{r_{\min }}, \quad Y=\frac{y}{r_{\min }}, \\
& \left\{f_{L}, g_{L}, h_{L}, f_{S}, h_{S}, \tau_{S}\right\}=\frac{r_{\min }^{2}}{D}\left\{k_{o f f}, k_{d e g}, k_{o n} R_{0}, j_{o f f}, j_{\text {on }} R_{0}, \tau\right\}, \\
& \{A, B, U, S\}=\frac{1}{R_{0}}\{[L],[L R],[L C],[C]\}, \\
& \left\{W_{C \min }, W_{C \max }, W_{\text {Corg }}, W_{L \min }, W_{L \max }, W_{L \operatorname{mat}}\right\}=\frac{r_{\min }^{2}}{D R_{0}}\left\{V_{C \min }, V_{C \max }, V_{C o r g}, V_{L \min }, V_{L m a x}, V_{L m a t}\right\}, \\
& \{P, Q\}=\frac{r_{\min }^{2}}{D}\{a, b\}, \\
& \left\{d_{L}, d_{L C}, d_{C}\right\}=\frac{1}{D}\left\{D_{L}, D_{L C}, D_{C}\right\}, \quad \overline{\gamma_{C}}=\gamma_{C} R_{0}, \quad \overline{\gamma_{L}}=\frac{\gamma_{L}}{R_{0}},
\end{aligned}
$$

where $D$ is the maximum of $D_{L}, D_{L C}$ and $D_{C}$ in (35).

In terms of the normalized quantities, the system (31) becomes to the following dimensionless form

$$
\left\{\begin{array}{l}
\frac{\partial A}{\partial T}=d_{L} \nabla^{2} A-h_{L} A(1-B)+f_{L} B-h_{S} A S+\left(f_{S}+\tau_{S}\right) U+W_{L} \\
\frac{\partial B}{\partial T}=h_{L} A(1-B)-\left(f_{L}+g_{L}\right) B \\
\frac{\partial U}{\partial T}=d_{L C} \nabla^{2} U+h_{S} A S-\left(f_{S}+\tau_{S}\right) U \\
\frac{\partial S}{\partial T}=d_{C} \nabla^{2} S-h_{S} A S+f_{S} U+W_{C}
\end{array}\right.
$$

where

$$
\begin{aligned}
& W_{C}=W_{C \min }+\frac{W_{C \max }-W_{C \min }}{1+\overline{\gamma_{C}} B}+ \begin{cases}W_{C o r g} e^{-P T}, & \text { if } X \geqslant \frac{7}{8} \\
0, & \text { otherwise. }\end{cases} \\
& W_{L}=W_{L \min }+\frac{W_{L \max }-W_{L \min }}{1+\bar{\gamma}_{L} B^{-1}}+W_{L m a t} e^{-Q T} .
\end{aligned}
$$

The non-dimensionalized parameters corresponding to those in (35) are:

$$
\begin{aligned}
& d_{L}=d_{L S}=d_{C}=1, \quad h_{L}=1.1068 \times 10^{3} \\
& f_{L}=3.6894 \times 10^{-3}, \quad h_{S}=2.7671 \times 10^{4} \\
& f_{S}=9.2235 \times 10^{-3}, \quad \tau_{S}=9.2235 \\
& g_{L}=0.4612, \quad W_{C \min }=0.24596 \\
& W_{C \max }=24.596, \quad W_{C o r g}=2.0538 \times 10^{2} \\
& \overline{\gamma_{C}}=30, \quad P=15.4032, \\
& W_{L \min }=3.0745 \times 10^{-3}, \quad W_{L \max }=1.8447 \\
& \overline{\gamma_{L}}=3.3333, \quad W_{L m a t}=15.4032, \quad Q=15.4032
\end{aligned}
$$

We use our new second order Krylov IIF method with $P^{1}$ DG spatial scheme to simulate the system (42)-(45), to the normalized final time $T=10$. The spatial triangular mesh is a much more refined one based on the mesh shown in Fig. 1(b). It has 154,560 triangular elements. The sparse matrix $\mathbf{A}$ has the huge size $463680 \times 463680$. The original IIF methods can not be applied to such big size problem directly on a regular computer. But our Krylov IIF method designed in this paper can simulate the system stably and efficiently. The time step size is taken to be $\Delta t=0.5 h_{\min }=6.416 \times 10^{-4}$ which is the advantage of the implicit methods for a parabolic problem. The simulation results of the normalized concentrations of morphogen molecules are shown in Fig. 2. The Fig. 2(b) shows a morphogen gradient of the BMP-receptor complex which induces the cell differentiation and tissue patterns. In the zebrafish dorsal-ventral patterning, the high concentration region of the BMPreceptor complex will develop into the ventral tissue, and the low concentration region of the BMP-receptor complex will develop into the dorsal tissue as shown in Fig. 2.

\section{Discussions and conclusions}

In this paper, we contributed two aspects in high order numerical methods. One is the development of a new Krylov subspace based implicit integration factor method for solving large ODE systems with both stiff linear and nonlinear terms, arising from numerical spatial discretization of time-dependent partial differential equations (PDEs) with linear high order 


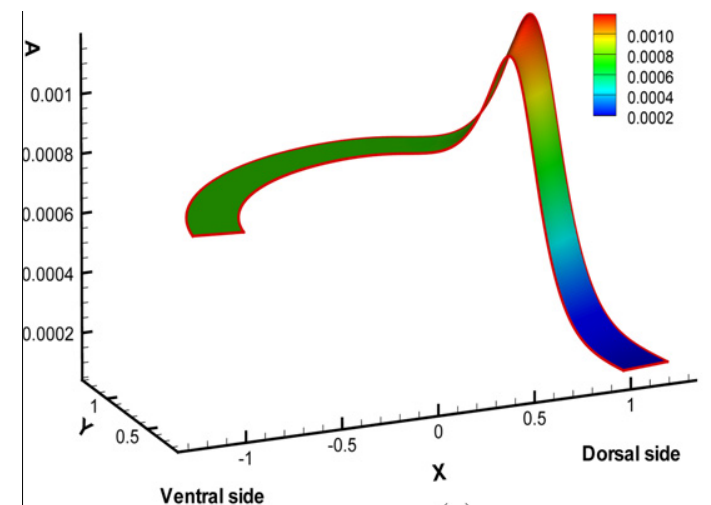

(a)

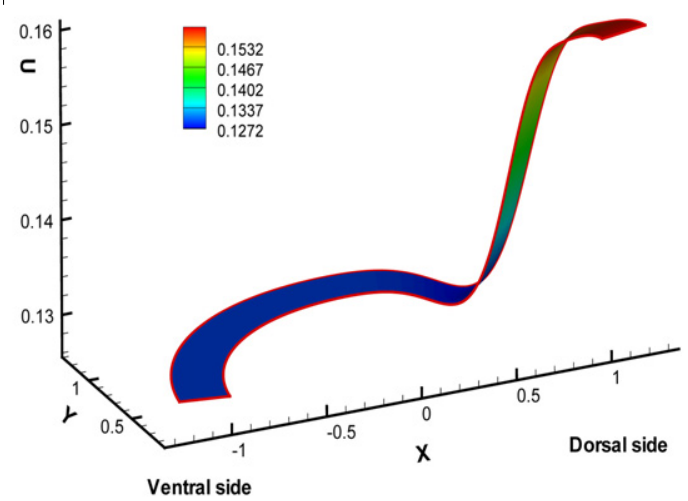

(c)

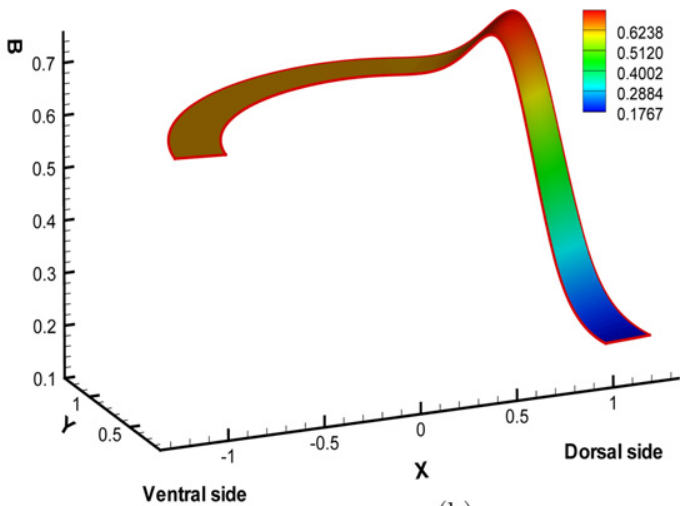

(b)

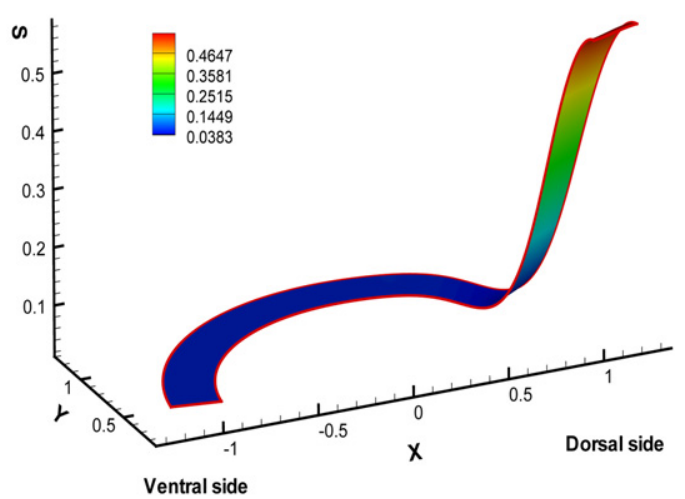

(d)

Fig. 2. Simulation results of the normalized concentrations of morphogens for the zebrafish system. Normalized final time $T=10$. The spatial triangular mesh has 154,560 elements. $\Delta t=0.5 h_{\min }=6.416 \times 10^{-4}$. (a) A: the normalized concentration of molecule BMP; (b) B: the normalized concentration of BMPreceptor complexes; (c) U: the normalized concentration of BMP-Chordin complexes; (d) S: the normalized concentration of molecule Chordin.

terms and stiff lower order nonlinear terms. The method can preserve the nice property of the original IIF scheme [48] that the exact evaluation of the linear part is decoupled from the implicit treatment of the nonlinear part. Furthermore, it can efficiently and accurately solve the large ODE systems arising from spatial discretization (e.g. DG methods) on high dimensional unstructured meshes for PDEs defined on complex geometrical domains. At the same time, this method provides an efficient and robust time discretization technique for DG methods on unstructured meshes for solving PDEs which have high order spatial derivatives. It is still an active area to develop efficient high-order accurate time-stepping methods for DG methods to solve PDEs with high order derivatives on multi-dimensional spatial domains. This is the second aspect in which this paper is trying to contribute.

By using numerical examples of solving reaction-diffusion PDEs with exact solutions and a realistic mathematical model in morphogenesis, we show the nice efficiency, stability and accuracy of the Krylov IIF-DG method. For this method, we can use a large time step size which is proportional to the spatial grid size to solve parabolic PDEs such as the reaction-diffusion equations. Numerical examples also show that the error generated by the Krylov subspace approximation with a quite small dimension ( the dimension $M=25$ for all mesh sizes) does not affect the accuracy orders of the IIF time discretizations ((5) or (6)) or the DG spatial discretizations (14), hence it is already much smaller than the DG spatial and IIF temporal truncation errors.

Because the computations of matrix exponential by the scaling and squaring method [30] are only needed for very small matrices ( $25 \times 25$ matrices in this paper), operations required with the original huge matrix are a few matrix-by-vector multiplications, which makes the algorithm easy to parallelize.

In this paper, we only used the numerical experiments to test the algorithm. The current ongoing work includes the analysis of the error generated by the Krylov subspace approximation and its effects on the truncation errors of the IIF and DG discretizations. The error analysis techniques in [26] will be used. In the numerical experiments of this paper, reaction-diffusion PDEs are used as examples. The Krylov IIF methods developed in this paper can be straightforwardly extended to solve more complex equations such as advection-reaction-diffusion PDEs and also applied to other DG methods such as the LDG methods $[65,63]$ for solving PDEs involving high order spatial derivatives on multi-dimensional unstructured meshes. These are our planned research at the next stage. 


\section{References}

[1] S. Adjerid, H. Temimi, A discontinuous Galerkin method for higher-order ordinary differential equations, Computer Methods in Applied Mechanics and Engineering 197 (2007) 202-218.

[2] U. Ascher, S. Ruuth, B. Wetton, Implicit-explicit methods for time-dependent PDE's, SIAM Journal of Numerical Analysis 32 (1995) 797-823.

[3] U.M. Ascher, S.J. Ruuth, R.J. Spiteri, Implicit-explicit Runge-Kutta methods for time-dependent partial differential equations, Applied Numerical Mathematics 25 (1997) 151-167.

[4] F. Bassi, S. Rebay, A high-order accurate discontinuous finite element method for the numerical solution of the compressible Navier-Stokes equations, Journal of Computational Physics 131 (1997) 267-279.

[5] G. Beylkin, J.M. Keiser, On the adaptive numerical solution of nonlinear partial differential equations in wavelet bases, Journal of Computational Physics 132 (1997) 233-259.

[6] G. Beylkin, J.M. Keiser, L. Vozovoi, A new class of time discretization schemes for the solution of nonlinear PDEs, Journal of Computational Physics 147 (1998) 362-387.

[7] R. Biswas, K.D. Devine, J. Flaherty, Parallel, adaptive finite element methods for conservation laws, Applied Numerical Mathematics 14 (1994) $255-283$.

[8] J.P. Boyd, Chebyshev and Fourier Spectral Methods, Dover, New York, 2001.

[9] A. Bourlioux, A.T. Layton, M.L. Minion, High-order multi-implicit spectral deferred correction methods for problems of reactive flow, Journal of Computational Physics 189 (2003) 651-675.

[10] C. Canuto, M.Y. Hussaini, A. Quarteroni, T.A. Zang, Spectral Methods in Fluid Dynamics, Springer Series in Computational Physics, Springer- Verlag, Berlin, 1988.

[11] Y. Cheng, C.-W. Shu, A discontinuous Galerkin finite element method for time dependent partial differential equations with higher order derivatives, Mathematics of Computation 77 (2008) 699-730.

[12] A. Christlieb, B. Ong, J.-M. Qiu, Integral deferred correction methods constructed with high order Runge-Kutta integrators, Mathematics of Computation 79 (2010) 761-783.

[13] B. Cockburn, Discontinuous Galerkin methods for convection-dominated problems, in: T.J. Barth, H. Deconinck (Eds.), High-Order Methods for Computational Physics, Lecture Notes in Computational Science and Engineering, vol. 9, Springer, 1999, pp. 69-224.

[14] B. Cockburn, S. Hou, C.-W. Shu, The Runge-Kutta local projection discontinuous Galerkin finite element method for conservation laws IV: the multidimensional case, Mathematics of Computation 54 (1990) 545-581.

[15] B. Cockburn, G. Karniadakis, C.-W. Shu, The development of discontinuous Galerkin methods, in: B. Cockburn, G. Karniadakis, C.-W. Shu (Eds.), Discontinuous Galerkin Methods: Theory, Computation and Applications, Lecture Notes in Computational Science and Engineering, vol. 11, Springer, 2000, pp. 3-50. Part I: Overview.

[16] B. Cockburn, S.-Y. Lin, C.-W. Shu, TVB Runge-Kutta local projection discontinuous Galerkin finite element method for conservation laws III: one dimensional systems, Journal of Computational Physics 84 (1989) 90-113.

[17] B. Cockburn, C.-W. Shu, TVB Runge-Kutta local projection discontinuous Galerkin finite element method for conservation laws II: general framework, Mathematics of Computation 52 (1989) 411-435.

[18] B. Cockburn, C.-W. Shu, The Runge-Kutta local projection P1-discontinuous Galerkin finite element method for scalar conservation laws, Mathematical Modelling and Numerical Analysis 25 (1991) 337-361.

[19] B. Cockburn, C.-W. Shu, The Runge-Kutta discontinuous Galerkin method for conservation laws V: multidimensional systems, Journal of Computational Physics 141 (1998) 199-224.

[20] B. Cockburn, C.-W. Shu, Runge-Kutta discontinuous Galerkin methods for convection-dominated problems, Journal of Scientific Computing 16 (2001) $173-261$.

[21] B. Cockburn, C.-W. Shu, The local discontinuous Galerkin method for time-dependent convection-diffusion systems, SIAM Journal on Numerical Analysis 35 (1998) 2440-2463.

[22] S.M. Cox, P.C. Matthews, Exponential time differencing for stiff systems, Journal of Computational Physics 176 (2002) $430-455$

[23] Q. Du, W. Zhu, Modified exponential time differencing schemes: analysis and applications, BIT, Numerical Mathematics 45 (2) (2005) 307-328.

[24] A. Dutt, L. Greengard, V. Rokhlin, Spectral deferred correction methods for ordinary differential equations, BIT 40 (2) (2000) $241-266$.

[25] B. Fornberg, T.A. Driscoll, A fast spectral algorithm for nonlinear wave equations with linear dispersion, Journal of Computational Physics 155 (1999) 456-467.

[26] E. Gallopoulos, Y. Saad, Efficient solution of parabolic equations by Krylov approximation methods, SIAM Journal on Scientific and Statistical Computing 13 (5) (1992) 1236-1264.

[27] G. Gassner, F. Lorcher, C.-D. Munz, A contribution to the construction of diffusion fluxes for finite volume and discontinuous Galerkin schemes, Journal of Computational Physics 224 (2007) 1049-1063.

[28] S. Gottlieb, C.-W. Shu, Total variation diminishing Runge-Kutta schemes, Mathematics of Computation 67 (1998) $73-85$

[29] S. Gottlieb, C.-W. Shu, E. Tadmor, Strong stability preserving high order time discretization methods, SIAM Review 43 (2001) 89-112.

[30] N.J. Higham, The scaling and squaring method for the matrix exponential revisited, SIAM Review 51 (4) (2009) 747-764.

[31] M. Hochbruck, C. Lubich, On Krylov subspace approximations to the matrix exponential operator, SIAM Journal on Numerical Analysis 34 (5) (1997) 1911-1925.

[32] M. Hochbruck, A. Ostermann, Explicit exponential Runge-Kutta methods for semilinear parabolic problems, SIAM Journal on Numerical Analysis 43 (3) (2005) 1069-1090.

[33] J. Huang, J. Jia, M. Minion, Arbitrary order Krylov deferred correction methods for differential algebraic equations, Journal of Computational Physics 221 (2) (2007) 739-760.

[34] A. Kanevsky, M.H. Carpenter, D. Gottlieb, J.S. Hesthaven, Application of implicit-explicit high order Runge-Kutta methods to discontinuous-Galerkin schemes, Journal of Computational Physics 225 (2) (2007) 1753-1781.

[35] A.-K. Kassam, L.N. Trefethen, Fourth-order time stepping for stiff PDEs, SIAM Journal on Scientific Computing 26 (4) (2005) $1214-1233$.

[36] C.A. Kennedy, M.H. Carpenter, Additive Runge-Kutta schemes for convection-diffusion-reaction equations, Applied Numerical Mathematics 44 (2003) 139-181.

[37] R.M. Kirby, G.E. Karniadakis, Selecting the numerical flux in discontinuous Galerkin methods for diffusion problems, Journal of Scientific Computing $(22-23)(2005)$ 385-411.

[38] S. Krogstad, Generalized integrating factor methods for stiff PDEs, Journal of Computational Physics 203 (2005) $72-88$.

[39] A. Lander, Q. Nie, F. Wan, Do morphogen gradients arise by diffusion?, Development Cell 2 (6) (2002) 785-796

[40] A. Lander, Q. Nie, F. Wan, Y.-T. Zhang, Localized ectopic expression of Dpp receptors in a Drosophila embryo, Studies in Applied Mathematics 123 (2009) 175-214.

[41] A.T. Layton, M.L. Minion, Conservative multi-implicit spectral deferred correction methods for reacting gas dynamics, Journal of Computational Physics 194 (2) (2004) 697-715

[42] A.T. Layton, M.L. Minion, Implications of the choice of predictors for semi-implicit Picard integral deferred corrections methods, Communications in Applied Mathematics and Computational Science 2 (1) (2007) 1-34.

[43] D. Levy, C.-W. Shu, J. Yan, Local discontinuous Galerkin methods for nonlinear dispersive equations, Journal of Computational Physics 196 (2004) 751772 .

[44] Y. Maday, A.T. Patera, E.M. Ronquist, An operator-integration-factor splitting method for time-dependent problems: application to incompressible fluid flow, Journal of Scientific Computing 5 (1990) 263-292. 
[45] M.L. Minion, Semi-implicit spectral deferred correction methods for ordinary differential equations, Communications in Mathematical Sciences 1 (3) (2003) 471-500.

[46] C. Moler, C. Van Loan, Nineteen dubious ways to compute the exponential of a matrix, twenty-five years later, SIAM Review 45 (2003) 3-49.

[47] H.N. Najm, P.S. Wyckoff, O.M. Knio, A semi-implicit numerical scheme for reacting flow. I. Stiff chemistry, Journal of Computational Physics 143 (1998) $381-402$.

[48] Q. Nie, Y.-T. Zhang, R. Zhao, Efficient semi-implicit schemes for stiff systems, Journal of Computational Physics 214 (2006) $521-537$.

[49] Q. Nie, F. Wan, Y.-T. Zhang, X.-F. Liu, Compact integration factor methods in high spatial dimensions, Journal of Computational Physics 227 (2008) 5238-5255.

[50] W.H. Reed, T.R. Hill, Triangular mesh methods for neutron transport equation. Technical Report LA-UR-73-479, Los Alamos Scientific Laboratory, 1973.

[51] S.J. Ruuth, Implicit-explicit methods for reaction-diffusion problems in pattern formation, Journal of Mathematical Biology 34 (1995) $148-176$.

[52] Y. Saad, Analysis of some Krylov subspace approximations to the matrix exponential operator, SIAM Journal on Numerical Analysis 29 (1) (1992) 209228.

[53] Y. Saad, Iterative Methods for Sparse Linear Systems, SIAM, 2003.

[54] C.-W. Shu, TVD time discretizations, SIAM Journal on Scientific and Statistical Computing 9 (1988) 1073-1084.

[55] C.-W. Shu, S. Osher, Efficient implementation of essentially non-oscillatory shock-capturing schemes, Journal of Computational Physics 77 (1988) 439471.

[56] L.N. Trefethen, Spectral Methods in Matlab, SIAM, 2000.

[57] L.N. Trefethen, D. Bau, Linear Algebra, SIAM, 1997.

[58] B. van Leer, S. Nomura, Discontinuous Galerkin for diffusion, in: 17th AIAA Computational Fluid Dynamics Conference. June 6-9, 2005, AIAA paper 2005-5108.

[59] J.G. Verwer, B.P. Sommeijer, W. Hundsdorfer, RKC time-stepping for advection-diffusion-reaction problems, Journal of Computational Physics 201 (2004) 61-79.

[60] Y. Xia, Y. Xu, C.-W. Shu, Efficient time discretization for local discontinuous Galerkin methods, Discrete and Continuous Dynamical Systems - Series B 8 (2007) 677-693.

[61] Y. Xu, C.-W. Shu, Local discontinuous Galerkin methods for three classes of nonlinear wave equations, Journal of Computational Mathematics 22 (2004) 250-274.

[62] Y. Xu, C.-W. Shu, Local discontinuous Galerkin methods for nonlinear Schrodinger equations, Journal of Computational Physics 205 (2005) $72-97$.

[63] Y. Xu, C.-W. Shu, Local discontinuous Galerkin methods for two classes of two dimensional nonlinear wave equations, Physica D 208 (2005) 21-58.

[64] Y. Xu, C.-W. Shu, Local discontinuous Galerkin methods for the Kuramoto-Sivashinsky equations and the Ito-type coupled KdV equations, Computer Methods in Applied Mechanics and Engineering 195 (2006) 3430-3447.

[65] J. Yan, C.-W. Shu, A local discontinuous Galerkin method for KdV type equations, SIAM Journal on Numerical Analysis 40 (2002) $769-791$.

[66] J. Yan, C.-W. Shu, Local discontinuous Galerkin methods for partial differential equations with higher order derivatives, Journal of Scientific Computing 17 (2002) 27-47.

[67] Y.-T. Zhang, A. Lander, Q. Nie, Computational analysis of BMP gradients in dorsal-ventral patterning of the zebrafish embryo, Journal of Theoretical Biology 248 (4) (2007) 579-589.

[68] X. Zhong, Additive semi-implicit Runge-Kutta methods for computing high-speed nonequilibrium reactive flows, Journal of Computational Physics 128 (1996) 19-31.

[69] J. Zhu, Y.-T. Zhang, S.A. Newman, M. Alber, Application of discontinuous Galerkin methods for reaction-diffusion systems in developmental biology, Journal of Scientific Computing 40 (2009) 391-418. 